\title{
Alfnoor: A Retrieval Simulation of the Ariel Target List
}

\author{
Q. Changeat ${ }^{1}$ (D) A. Al-Refaie ${ }^{1}$ (D), L. V. Mugnai ${ }^{2}$ (D), B. Edwards ${ }^{1}$ (D), I. P. Waldmann ${ }^{1}$ (D), E. Pascale ${ }^{2}$, and G. Tinetti ${ }^{1}$ (DD \\ ${ }^{1}$ Department of Physics and Astronomy University College London Gower Street, WC1E 6BT London, UK; quentin.changeat.18@ucl.ac.uk \\ 2 Dipartimento di Fisica La Sapienza Universita di Roma Piazzale Aldo Moro 2, I-00185 Roma, Italy \\ Received 2020 February 29; revised 2020 June 2; accepted 2020 June 5; published 2020 July 27
}

\begin{abstract}
In this work, we present Alfnoor, a dedicated tool optimized for population studies of exoplanet atmospheres. Alfnoor combines the latest version of the retrieval algorithm, TauREx 3, with the instrument noise simulator ArielRad and enables the simultaneous retrieval analysis of a large sample of exo-atmospheres. We applied this tool to the Ariel list of planetary candidates and focus on hydrogen dominated, cloudy atmospheres observed in transit with the Tier-2 mode (medium Ariel resolution). As a first experiment, we randomized the abundancesranging from $10^{-7}$ to $10^{-2}$-of the trace gases, which include $\mathrm{H}_{2} \mathrm{O}, \mathrm{CH}_{4}, \mathrm{CO}, \mathrm{CO}_{2}$, and $\mathrm{NH}_{3}$. This exercise allowed us to estimate the detection limits for Ariel Tier- 2 and Tier-3 modes when clouds are present. In a second experiment, we imposed an arbitrary trend between a chemical species and the effective temperature of the planet. A last experiment was run requiring molecular abundances being dictated by equilibrium chemistry at a certain temperature. Our results demonstrate the ability of Ariel Tier-2 and Tier-3 surveys to reveal trends between the chemistry and associated planetary parameters. Future work will focus on eclipse data, on atmospheres heavier than hydrogen, and will be applied also to other observatories.
\end{abstract}

Unified Astronomy Thesaurus concepts: Space telescopes (1547); Exoplanet atmospheric composition (2021); Transmission spectroscopy (2133)

\section{Introduction}

In the last decade, the field of extra-solar planets has very rapidly grown and matured. The NASA Kepler mission and other dedicated surveys from the ground have revolutionized our understanding of these extraterrestrial worlds. We are now aware of the ubiquity and vast diversity of planets outside our solar system, ranging from ultra-hot giant planets (Cameron et al. 2010; Delrez et al. 2016; Gaudi et al. 2017) to more temperate Earths and Super-Earths (Gillon et al. 2016; Ment et al. 2019). With the Transiting Exoplanet Survey Satellite (TESS; Ricker et al. 2014), Gaia (Gaia Collaboration et al. 2016), CHaracterizing ExoPlanet Satellite (CHEOPS; Broeg et al. 2013), Spectro-Polarimetic High contrast imager for Exoplanets REsearch (SPHERE; Beuzit et al. 2019), Gemini Planet Imager (GPI; Macintosh et al. 2014), and Echelle SPectrograph for Rocky Exoplanets and Stable Spectroscopic Observations (ESPRESSO; Pepe et al. 2010) currently operating and space missions like PLAnetary Transits and Oscillations of stars (PLATO; Rauer et al. 2016) and Wide Field Infrared Survey Telescope (WFIRST; Bennett et al. 2018) soon to come online, the statistics of planets in our galaxy will evolve even further in the next decade.

Current studies of exoplanetary atmospheres have been largely conducted using general observatories from spaceHubble Space Telescope and Spitzer Space Telescope-or from the ground-e.g., Very Large Telescope (VLT) CRyogenic high-resolution InfraRed Echelle Spectrograph (Crires), NASA Infrared Telescope Facility (NASA IRTF), Telescopio Nazionale Galileo (TNG), VLT-SPHERE, Gemini-GPI, Subaru -and thus results are often sparse and only available for a limited number of the discovered planets. As a result, most atmospheric retrieval studies have focused so far on the analysis of individual planets (Kreidberg et al. 2014; Line et al. 2016; Tsiaras et al. 2016, 2019) with only a few papers having attempted a consistent spectral analysis of multiple targets
(Sing et al. 2015; Barstow et al. 2016; Tsiaras et al. 2018; Pinhas et al. 2019). In the next decade, a new generation of observatories from space and the ground and dedicated missions (Gardner et al. 2006; Gilmozzi \& Spyromilio 2007; Skidmore 2015; Tinetti et al. 2018; Edwards et al. 2019b) will come online, offering a broader spectral coverage, higher signal-to-noise ratio $(\mathrm{S} / \mathrm{N})$, and the ability to study a significantly larger number of targets. The ESA-Ariel mission alone has been designed to deliver transit, eclipse, and phasecurve spectra for hundreds of planets, providing, for the first time, the chance to conduct a statistically significant survey of exoplanet atmospheres (Edwards et al. 2019a).

In most fields of astronomy (supernovae, brown dwarfs, black holes), revolutions in our understanding of the main processes often came from the study of the statistical behavior using large samples as opposed to individual studies. As the next generation of space telescopes come online, we will reach this important step for exo-atmospheres and it is therefore critical to be aware of the challenges associated with large-scale studies.

In this paper, we describe our integrated algorithm, Alfnoor, which combines the open source atmospheric retrieval code TauREx 3 (Al-Refaie et al. 2019) and the Ariel noise simulator ArielRad (Mugnai et al. 2020) with the aim to facilitate the spectral analysis and interpretation of populations of exoplanetary atmospheres (Section 2). Current Ariel's strategy is to observe planets in accordance to a four tier structure, where the aim of the second tier (Tier 2) of observations is to extract the key atmospheric constituents (Edwards et al. 2019a). In this paper we simulated Ariel Tier-2 and Tier-3 performances for a large sample of planets provided in Edwards et al. (2019a). For the selected targets, different, randomized atmospheric compositions were assumed and an automated retrieval analysis for each planet was performed. We then compared and discussed the results of the posterior distributions, as provided by the retrievals, to the ground truth to assess Ariel's ability to recover 
Table 1

Wavelength Coverage $(\lambda)$ and Resolutions $(R)$ of Each Spectrometer (NIRSpec, AIRS-CH0, and AIRS-CH1) for the Ariel Tiers

\begin{tabular}{lcccc}
\hline \hline Instrument & $\lambda(\mu \mathrm{m})$ & $R$-Tier 1 & $R$-Tier 2 & $R$-Tier 3 \\
\hline VISPhot & $0.5-0.6$ & $\varnothing$ & $\varnothing$ & $\varnothing$ \\
FGS1 & $0.6-0.8$ & $\varnothing$ & $\varnothing$ & $\varnothing$ \\
FGS2 & $0.8-1.1$ & $\varnothing$ & $\varnothing$ & $\varnothing$ \\
NIRSpec & $1.1-1.95$ & 1 & 10 & 20 \\
AIRS-CH0 & $1.95-3.9$ & 3 & 50 & 100 \\
AIRS-CH1 & $3.9-7.8$ & 1 & 10 & 20 \\
\hline
\end{tabular}

Note. We also show the photometers (VISPhot, FGS1, and FGS2).

accurately and precisely the abundances of the key trace gases and identify arbitrary injected chemical trends (Section 3). Finally we discuss these results in light of new facilities coming online soon and the next steps needed to progress further in our understanding of population studies (Section 4).

\section{Methodology and Software Description}

\subsection{Description of the Software}

To study large samples of exoplanetary spectra, we built a new tool: Alfnoor. Alfnoor combines the highly flexible nextgeneration retrieval code TauREx 3 with the ArielRad noise simulator to provide a unique framework dedicated to the study of exoplanetary populations with Ariel.

TauREx 3 (Al-Refaie et al. 2019) is the new version of TauREx (Waldmann et al. 2015a, 2015b). This complete rewrite takes the form of a library and is designed to make customization and external code integration easy. It uses the highly accurate line lists from the ExoMol (Tennyson et al. 2016), the high-resolution transmission molecular absorption database (HITRAN; Rothman \& Gordon 2014), and the hightemperature molecular spectroscopic database (HITEMP; Gordon et al. 2016) databases to build forward and retrieval models. A large number of options are available in terms of forward models (transmission, emission), chemical profiles (constant as a function of pressure, two-layer, equilibrium chemistry), temperature profiles (isothermal, NPoints; Guillot 2010), and cloud parameterizations (Gray; Bohren \& Huffman 2008; Lee et al. 2013).

ArielRad (Mugnai et al. 2020) estimates Ariel performances to observe a certain target when stellar, planetary, and orbital parameters are specified. It also calculates the required number of observations to match the requirements for each of Ariel's tiers (Edwards et al. 2019a). In our study we focused on Tier-2 observations, which are the core of the mission, and aim at characterizing the key chemical species, thermal structure, and the cloud properties of the selected atmospheres. Ariel observations are expected to cover the wavelengths from 0.5 to $7 \mu \mathrm{m}$. The telescope has three photometers: a visible photometer (VISPhot) and two fine guidance sensors (FGS1 and FGS2) that are also used for the observations. The telescope also has two spectrometers: the Near Infrared Spectrometer (NIRSpec) and the Ariel Infrared Spectrometer (AIRS). The resolution of the spectrometers is adapted to the tier levels. A description of the resolution achieved for each tier can be found in Tinetti et al. (2018), Edwards et al. (2019a), and Mugnai et al. (2020). It is summarized in Table 1.

The function Alfnoor-forward simulated high-resolution transit spectra with TauREx 3 for all of the targets. Next it
Table 2

List of Opacities Used in This Work

\begin{tabular}{ll}
\hline \hline Opacity & References \\
\hline $\mathrm{H}_{2}-\mathrm{H}_{2}$ & Abel et al. (2011), Fletcher et al. (2018) \\
$\mathrm{H}_{2}-\mathrm{He}$ & Abel et al. (2012) \\
$\mathrm{H}_{2} \mathrm{O}$ & Barton et al. (2017), Polyansky et al. (2018) \\
$\mathrm{CH}_{4}$ & Hill et al. (2013), Yurchenko \& Tennyson (2014) \\
$\mathrm{CO}$ & Li et al. (2015) \\
$\mathrm{CO}_{2}$ & Rothman et al. (2010) \\
$\mathrm{NH}_{3}$ & Yurchenko et al. (2011), Tennyson \& Yurchenko (2012) \\
\hline
\end{tabular}

called ArielRad to calculate the Ariel error bars, wavelength bins, and the number of required observations to reach Tier-2 performances for all the targets. The function alfnoor-inverse took the Tier-2 spectra generated by alfnoor-forward and performed atmospheric retrievals using TauREx 3 in fitting mode.

Tier-1 observations are studied in detail in L. Mugnai et al. (2020, in preparation). Our sample of planets consists of the 146 planets observed in transit at Tier 2 from the mission reference sample presented in Edwards et al. (2019a). Of these planets, 14 of them qualify for observations in Tier 3. The simulated planets are built to represent the entire parameter space. In our sample 20 planets have radius smaller than $2 R_{\mathrm{E}}$, 29 are between 2 and $5 R_{\mathrm{E}}$, and 97 have radius $>5 R_{\mathrm{E}}$. For a more detailed description of the methodology used to build this target list, we refer the reader to Edwards et al. (2019a). Future studies will concentrate on eclipse observations and/or secondary atmospheres.

\subsection{Approach and Initial Setups}

In all the models, the atmosphere is composed of $\mathrm{H}_{2}$ and $\mathrm{He}$ with a ratio of $\mathrm{He} / \mathrm{H}_{2}=0.17$. For the trace gases, the list and sources of the opacities used in this paper are presented in Table 2. Collision-induced absorption for $\mathrm{H}_{2}-\mathrm{H}_{2}$ and $\mathrm{H}_{2}-\mathrm{He}$ and Rayleigh scattering are included. For the retrievals, unless specified otherwise, we used the same assumptions: mixing ratios constant with pressure, temperature constant with pressure, and gray opaque clouds. While temperature variations with altitude are crucial for eclipse observations, in the case of transmission spectra, most studies assume isothermal temperature profiles. This is justified by the narrow wavelength coverage and $\mathrm{S} / \mathrm{N}$ in the available observations (with the Hubble Space Telescope), which does not allow the probing of large pressure regions in the planet atmosphere. The temperature variations in transmission act as a second-order parameter and the spectrum is most sensitive to the mean temperature value, which directly appears in the scale height. However, Barstow et al. (2012), Rocchetto et al. (2016), and Changeat et al. (2019a) highlighted the impact of temperature variations for high $\mathrm{S} / \mathrm{N}$ and broad wavelength coverage cases, indicating that the James Webb Space Telescope (JWST) and Ariel would be able to retrieve more complex temperature structures from transit spectra. As this study focuses on the capabilities of Ariel to recover chemical species, we do not investigate further the impact of non-isothermal temperature structures. We however note that this assumption could introduce biases to our results. Parameters that are traditionally determined using external methods are fixed to the true values, e.g., stellar radius, planetary mass, and $\mathrm{He} / \mathrm{H}_{2}$ ratio. The list of free parameters along with the priors used are described in Table 3 
Table 3

List of the Fit Parameters and Their Priors for the Retrievals (Alfnoor-inverse)

\begin{tabular}{|c|c|c|}
\hline Parameters & Priors & Scale \\
\hline Radius $\left(R_{\mathrm{J}}\right)$ & $\pm 50 \%$ & Linear \\
\hline Cloud pressure (bar) & $10-10^{-7}$ & $\log$ \\
\hline$T(\mathrm{~K})$ & $\pm 50 \%$ & Linear \\
\hline $\mathrm{H}_{2} \mathrm{O}(\mathrm{VMR})$ & $10^{-12}-10^{-1}$ & $\log$ \\
\hline $\mathrm{CH}_{4}(\mathrm{VMR})$ & $10^{-12}-10^{-1}$ & $\log$ \\
\hline $\mathrm{CO}$ (VMR) & $10^{-12}-10^{-1}$ & $\log$ \\
\hline $\mathrm{CO}_{2}(\mathrm{VMR})$ & $10^{-12}-10^{-1}$ & $\log$ \\
\hline $\mathrm{NH}_{3}(\mathrm{VMR})$ & $10^{-12}-10^{-1}$ & $\log$ \\
\hline
\end{tabular}

Note. We take a conservative approach and select larger bounds than the ones used to randomly generate the planets in forward mode. The chemical abundances are expressed in vertical mixing ratios (VMR).

In this study, we aim to explore two particular aspects of the Ariel mission:

1. the ability of Ariel to detect molecular species and the detection limits for these molecules in the context of cloudy primary atmospheres observed in transit. This task can be easily achieved by performing retrievals on an unbiased data set of planets where the atmospheric composition is randomized and by assessing the cases that have been successfully recovered.

2. the ability of Ariel to reveal chemical trends in exoplanet populations. To assess this possibility, a biased sample can be used as an input where an artificial trend is introduced.

We describe below the actual implementation of this plan.

1. Unbiased sample. We built the forward model using the stellar and planetary basic parameters from Edwards et al. (2019a) for the Ariel target list. We randomized the chemistry, temperature, and cloud parameters so that a unique set of these parameters is adopted for each planet of each sample. For the chemistry, we considered constant profiles with pressure for the mixing ratios of $\mathrm{H}_{2} \mathrm{O}, \mathrm{CH}_{4}$, $\mathrm{CO}, \mathrm{CO}_{2}$, and $\mathrm{NH}_{3}$ and chose a random abundance in logarithmic scale from $10^{-7}$ to $10^{-2}$. For clouds, we generated gray opaque clouds with random top pressures varying in log-scale from 10 bar (equivalent to no clouds) to $10^{-3}$ bar. Finally, the atmospheric temperatures were also randomly generated and allowed to assume values between $0.7 \times T_{\text {eff }}$ and $1.05 \times T_{\text {eff }}$, where $T_{\text {eff }}$ is the effective temperature in the Ariel target list of Edwards et al. (2019a). The temperature was consciously selected biased toward lower values to account for differences between effective temperature and the terminator temperature (Caldas et al. 2019). We repeated the generation of the observed spectra twice to build unscattered and scattered data sets. In the unscattered set, we conserve the theoretical simulated spectra as is. As scatter generally arises from the random realization of observations, we apply a Gaussian scatter to a second data set using the true value as mean and the simulated noise as variance. This scattered data set better describes what would be obtained in an actual observation by the telescope but cannot be used to characterize retrieval biases as unfortunate runs could lead to large discrepancies between true and retrieved values (Feng et al. 2018; Changeat et al. 2019a). Unscattered spectra are more suitable for the study of retrieval biases and intrinsic correlations between the atmospheric parameters (Feng et al. 2018). On the opposite, scattered spectra can inform us on the stability and the redundancy in the information content of Ariel spectra. Previous studies have used both types to simulate observations by future telescopes (Barstow et al. 2012; Tinetti et al. 2015, 2018; Feng et al. 2016, 2018; Rocchetto et al. 2016; Batalha et al. 2017; Mollière et al. 2017; Blumenthal et al. 2018; Changeat et al. 2019a, 2019b; Edwards et al. 2019b; Lustig-Yaeger et al. 2019). Feng et al. (2018) results predicted that the retrieved uncertainties should be similar in both scattered and unscattered runs but that the retrieved mean could be different. Here, we use our two data sets to investigate these predictions keeping in mind that if Ariel spectra contain enough information content redundancy, we should not see large differences in the retrieved mean values.

2. Biased samples. We imposed first a linear relationship between the logarithmic abundance of water and the temperature. We enacted this correlation water temperature by requiring a mixing ratio of $10^{-4}$ for an effective temperature of $1000 \mathrm{~K}$ and $10^{-3}$ for an effective temperature of $2000 \mathrm{~K}$.

We then tried a more realistic example where the atmospheres were assumed to be in chemical equilibrium and simulated accordingly the chemical abundances and profiles (Agúndez et al. 2012). We used the same solar $\mathrm{C} / \mathrm{O}$ ratio and metallicity for all the planets in the sample. To recover the input profiles, we used in the retrievals both free, constant with altitude chemical profiles and profiles that are forced to follow chemical equilibrium prescriptions. We did not test the entire sample with the two-layer chemistry retrieval scheme as presented in Changeat et al. (2019a), but we have run an example to show the expected improvements of this scheme over the pressure constant chemical profiles.

\section{Results}

\subsection{Unbiased Sample}

We show in Figure 1 both the observed and retrieved spectra for a subset of the simulated Ariel Tier- 2 observations, along with the correlation map between water abundance and temperature with their $1 \sigma$ uncertainties. The distance between the true and the retrieved value is visualized by the color of the point. The retrieved parameters are represented by the median chemical or temperature profiles weighted by the contribution function. The contribution function is defined as the wavelength averaged variations of the optical depth with pressure. This choice ensures that the values reported well reflect the conditions in the atmospheric regions probed by observations. In order to better visualize the Ariel detection limits in Tier 2, we also provide complementary plots of the retrieved abundances versus their true values for each molecule. The $\mathrm{H}_{2} \mathrm{O}$ map is presented in Figure 2.

The water-temperature map in Figure 1 clearly shows that our unbiased population is randomly spread in the parameter space, as expected. The retrieved temperature is very precise across the whole parameter space, showcasing the ability of Ariel Tier 2 to study a wide range of planets. It also illustrates that the retrieved values are mostly accurate for water abundances higher than $10^{-6}$; with the exception of a few cases, the retrieved values for water and temperature fall well 

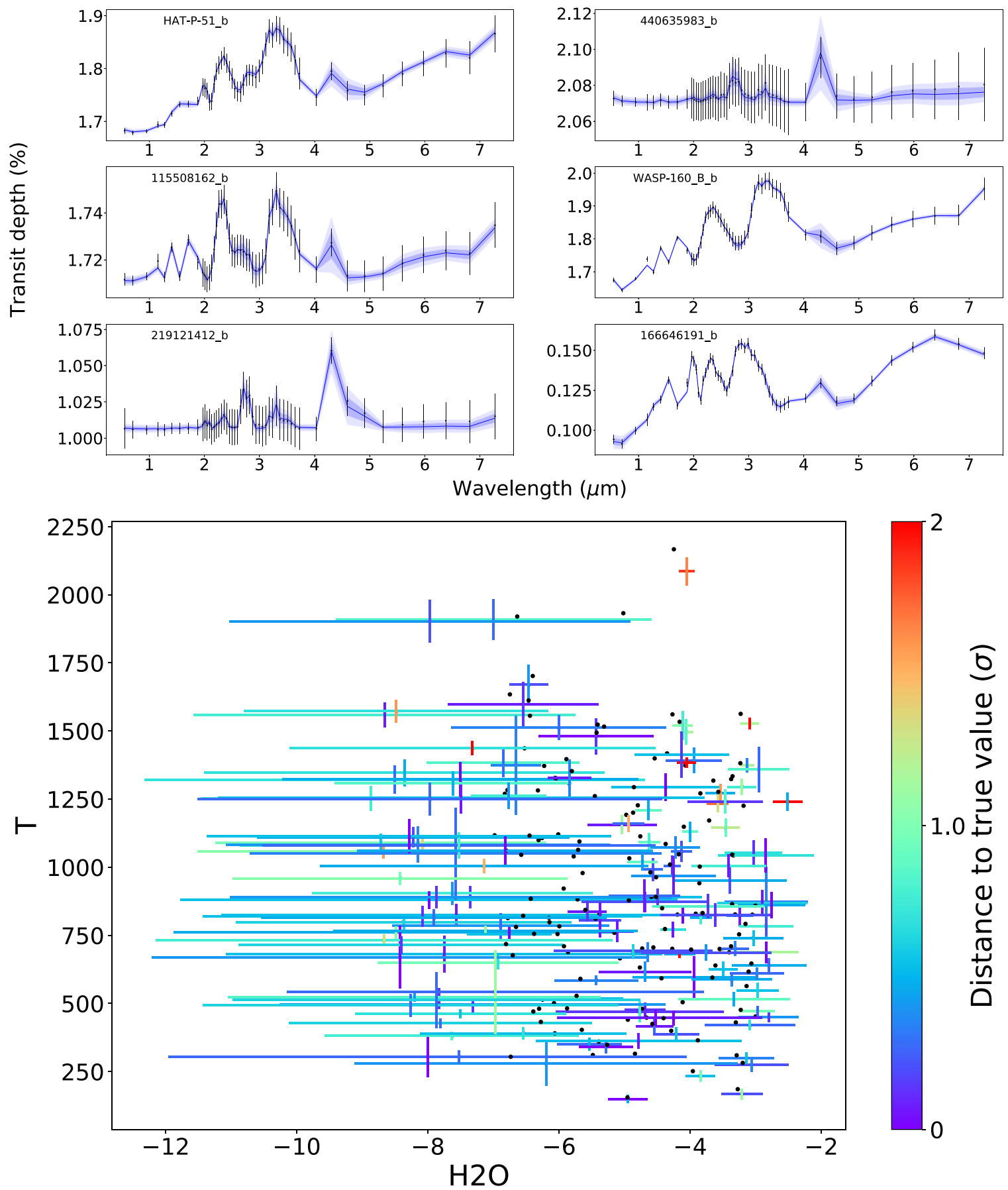

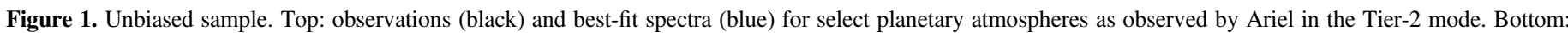

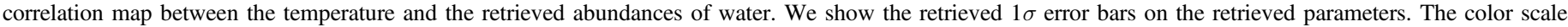
represents the distance to the true value (indicated with the black dots) in units of $1 \sigma$.

within the $1 \sigma$ error bars (blue to green in the color scale). We notice for water a rapid change in the posteriors for abundances smaller than $10^{-5}$, marked by large error bars on the left side of the plot. Indeed, when the abundance is too low, the retrievals are not able to distinguish well the features and provide only upper limits. This is an expected behavior and an indication of the Ariel detection limit for our sample of planets. This exercise was repeated for other molecules to assess Ariel ability to detect different sets of molecules in Tier- 2 mode. Other temperature-molecule maps, as well as the radius-cloud map, are reported in the Appendix (Figures A1-A5).
The detection limits are best visualized in the retrieved versus true abundances (see Figure 2). In the same figure, we also show the retrieved uncertainties versus input abundances as this allows us to distinguish three regimes. The first regime corresponds to low abundances where molecular detections are not possible; for example, between $10^{-7}$ and $10^{-6}$ for water no detections seem possible with Ariel. Other molecules are presented in Figure A6: $\mathrm{CH}_{4}$; Figure A7: CO; Figure A8: $\mathrm{CO}_{2}$; and Figure A9: $\mathrm{NH}_{3}$. It is interesting to note that when the molecules are not detected, the retrieved errors $(\sigma)$ are dominated by the size of the priors and the location of the 

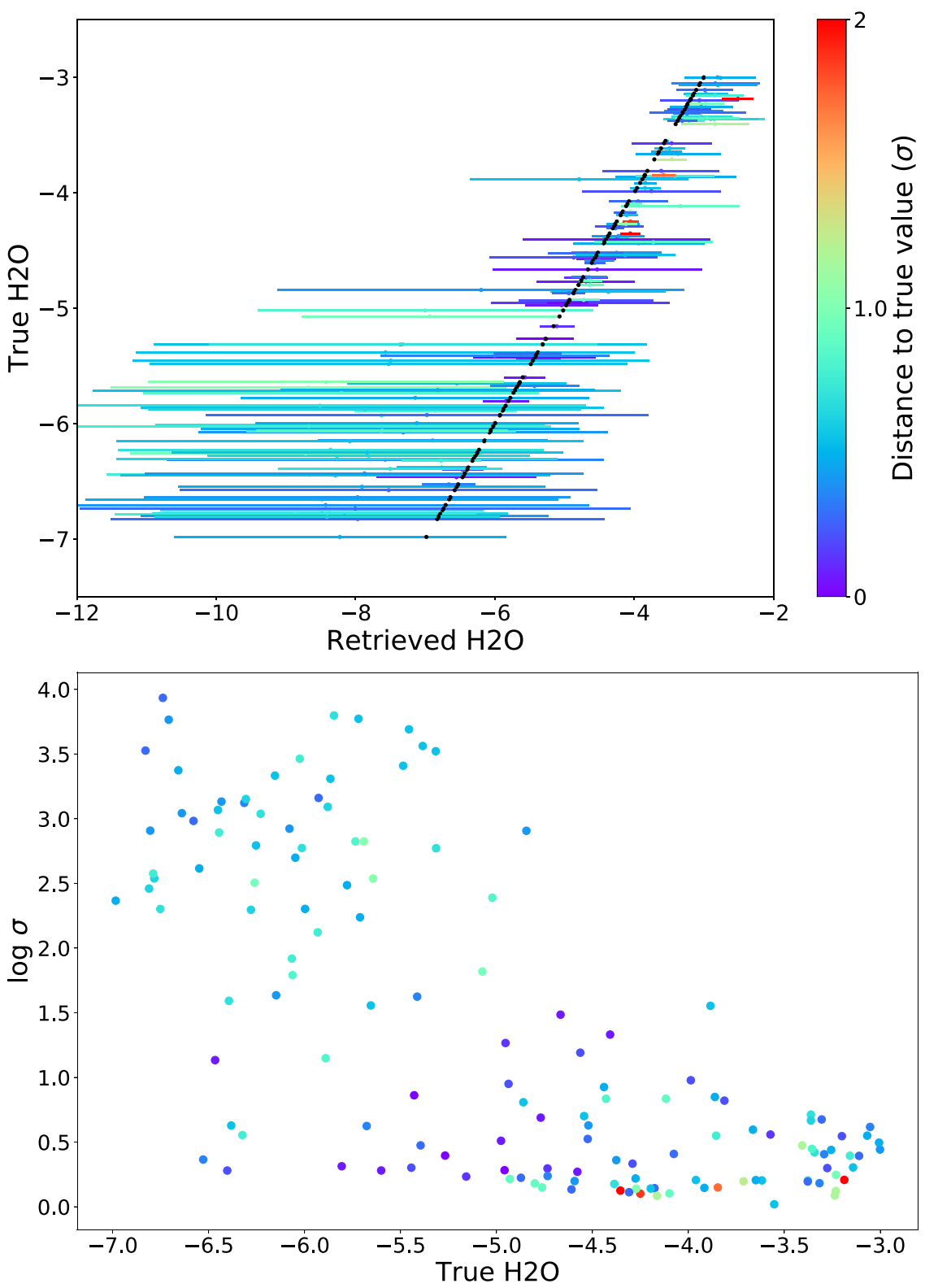

Figure 2. Map of the $\mathrm{H}_{2} \mathrm{O}$ retrieved abundance vs. the true value for the unbiased sample. The color scale of the $1 \sigma$ retrieved error bars represents the distance to the true value in units of $1 \sigma$.

detection limit: for water non-detection errors are between 2 and 4 orders of magnitude. The second regime for intermediate abundances displays a mix between successful detections and lack of evidence for the molecules. This corresponds to the region with large ranges in the retrieved errors (between $10^{-6}$ and $10^{-5}$ for water). In general, this variability is due to the other constituents in the planet that are susceptible to mask the signal of interest (e.g., clouds, other molecules). Finally, for the highest abundances, the retrieved uncertainties are low (less than 1 order of magnitude in the mixing ratios), which indicate that these abundances are always retrieved, regardless of the other constituents in the atmosphere.

Additionally, the map exploring the correlation between planetary radius and cloud top pressure shows that Ariel can separate well these parameters, most likely thanks to the FGS optical channels.

We repeated the same experiment with the second run composed of scattered spectra. Each planet is simulated with a new set of randomized parameters. As previously stated, the observed values of the transit depth are assumed to follow a normal distribution (the mean is the simulated transit depth and the standard deviation is the instrumental noise), which better reproduces a real observation. Figure 3 shows the watertemperature map. The other chemical parameters are reported in the Appendix (Figures A1-A5). From the analysis of the scattered spectra, we appreciate that the scattering of the data points around their true value does not necessary introduce biases in Ariel Tier-2 retrieval studies. Indeed, this result, which has already been explored in Feng et al. (2018) and Changeat et al. (2019a), naturally arises from the redundancy of the information relative to each molecule in the Ariel spectra and the fact that in most cases $N$ repeated observations are needed to obtain Tier- 2 requirements, therefore reducing by $1 / \sqrt{N}$ the scattering amplitude around their true value. Feng et al. (2018) highlighted that to avoid potential biases arising 

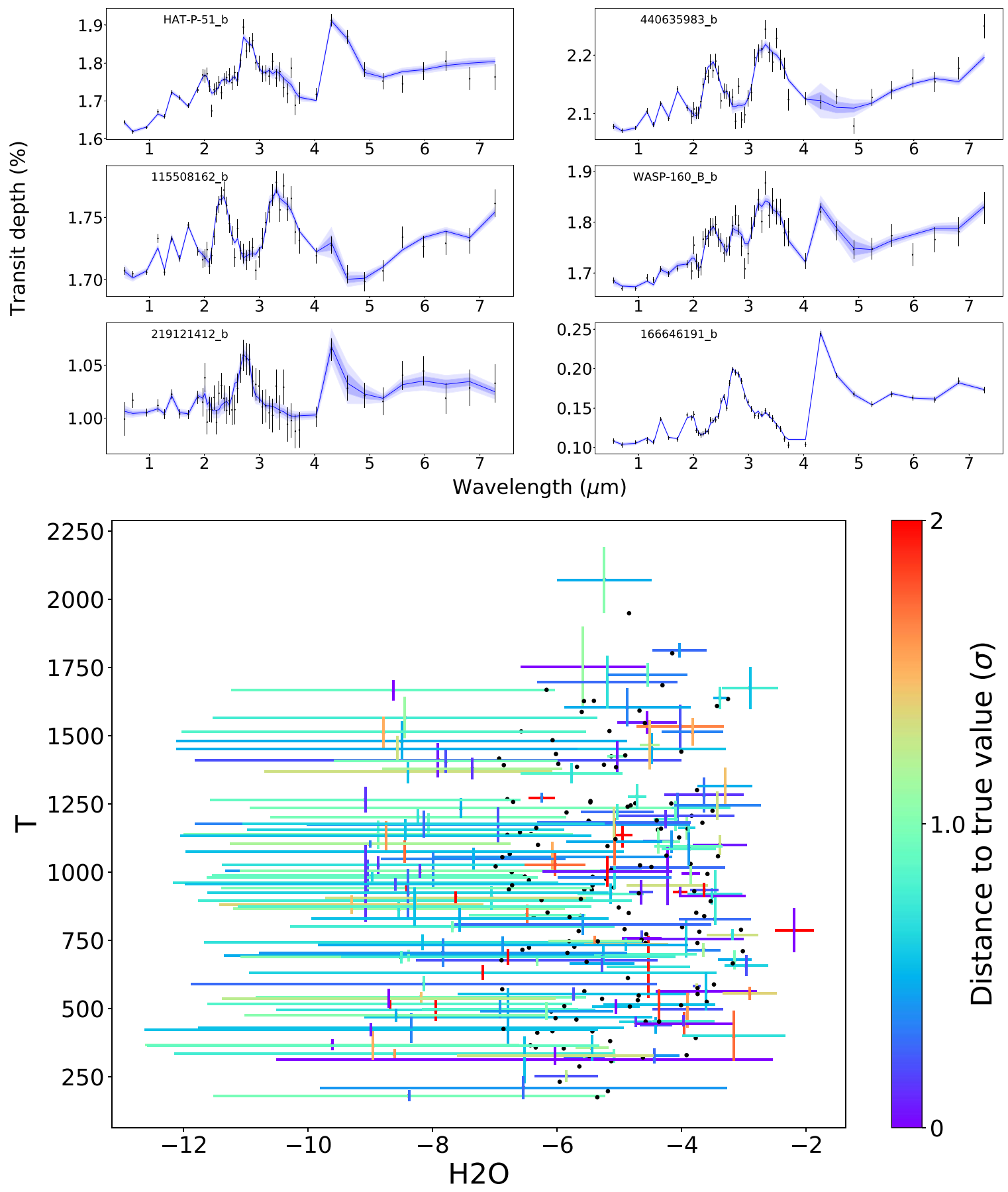

Figure 3. Same as Figure 1 but in this new run we scatter the Ariel spectra around their true values.

from individual noise instances one would essentially have to produce multiple retrievals with different noise instances and average the obtained results. As this was not computationally feasible, they chose not to scatter the spectra and use the true value as an approximation, stating that the shape of the posteriors would be accurate but that the position may be optimistically centered. For Ariel Tier-2 observations, the information content of the spectra is redundant enough to ensure that the retrieved values are not affected by this phenomenon and these are mostly centered around the true value in both scattered and nonscattered scenarios. For all molecules, we find that the correlation maps are very similar in both cases and the detection limits remain unchanged from the nonscattered runs. For the clouds, however, we note an overall increase in the distance to the true value (see correlation map in the Appendix, Figure A5). We note that in the simulations presented here, we considered fully opaque gray cloud cover, which is essentially the worst case scenario as no cloud features are detectable and it is well known to be degenerate with radius (Changeat et al. 2019b). More realistic cloud simulations will be considered in a future paper to test more thoroughly this case.

We summarize in Table 4 the approximate detection limits for each molecule considered. These represent the regions 


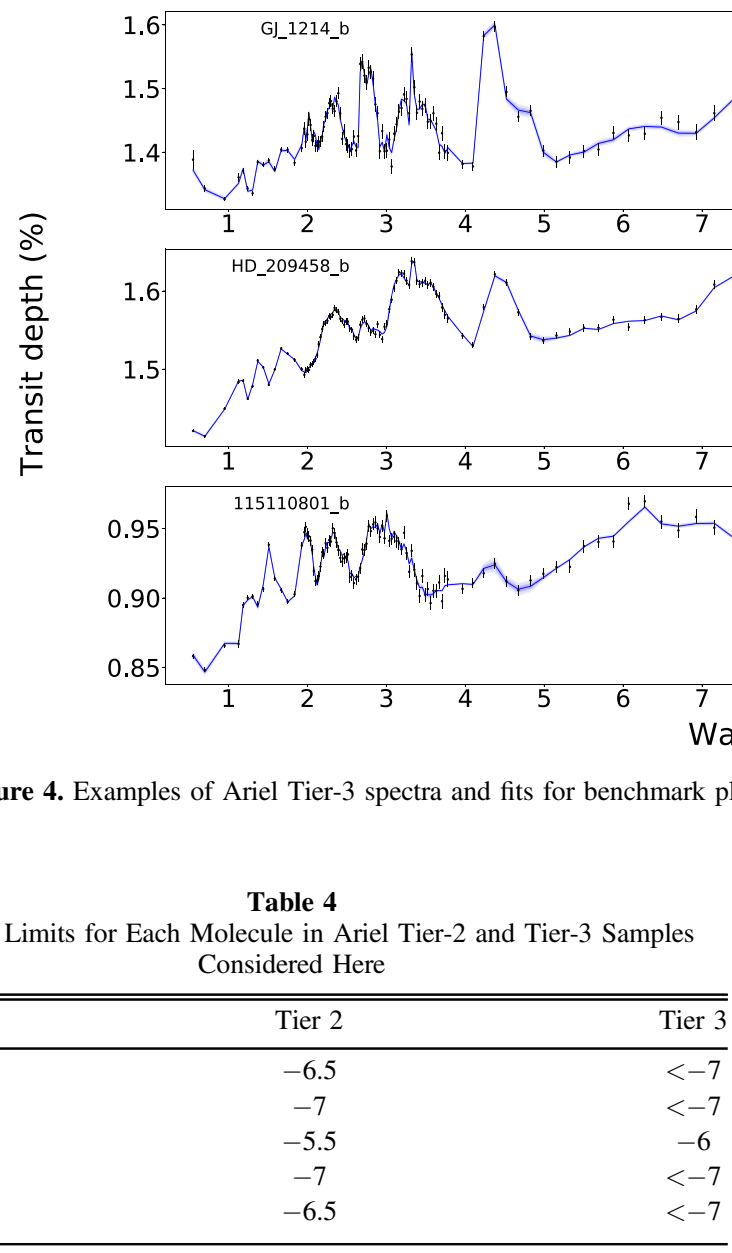

Note. The detection limits correspond to the lowest value we were able to extract abundance with less than 1 order of magnitude errors. The Tier-3 sample includes only 14 planets.

where our retrieval analysis have been able to extract constraints on the given atmospheric constituents. The stated detection limit corresponds to the lowest value that was successfully recovered with less than 1 order of magnitude uncertainties. As seen before, three regions of the parameter space can be identified: region 1 with no possible detections of the molecule, region 2 with detections depending on the other atmospheric properties, and region 3 where the molecule is always detected. This means the stated values do not represent a guaranty of detection, but rather the lowest limit we can hope to detect the molecule. In addition, we plot the contribution of each molecule individually in Figure A10 (each spectrum only contains $10^{-5}$ of the considered molecule) to show that the features span by each molecule. In general, Ariel Tier- 2 spectra should enable molecular detections down to mixing ratios of $10^{-6}$. In our simulations, only $\mathrm{CO}$ appears to be difficult to detect at abundances smaller than $10^{-4}$. CO presents two features that are overlapping with $\mathrm{CO}_{2}$ at $4.5 \mu \mathrm{m}$ and with $\mathrm{CH}_{4}$ at $2.5 \mu \mathrm{m}$ and are relatively weak. In a real scenario (equilibrium chemistry), we believe $\mathrm{CO}$ could be more easily distinguishable as our unbiased assumption underestimates the $\mathrm{CO}$ abundance and overestimates the $\mathrm{CO}_{2}$ abundance by design (Agúndez et al. 2012; Venot et al. 2012; Venot \& Agúndez 2015). We also note that $\mathrm{H}_{2} \mathrm{O}$ and $\mathrm{CH}_{4}$ have a large number of anticorrelated features, which may give rise to more featureless spectra when the two molecules are present. For all parameters, Ariel Tier- 2 spectra provide accurate and precise estimates, as most of the retrieved error bars are less than $1 \sigma$ away from the true value. This statement applies to both nonscattered and scattered spectra.

For completeness, we performed additional retrievals for 14 benchmark planets in Tier-3 mode (Edwards et al. 2019a). The benchmark planets achieve a high $\mathrm{S} / \mathrm{N}$ in a very limited number of transits and are re-observed at different times to allow for temporal and spatial variability studies. In the examples presented here, we combined five transit observations to reach the required $\mathrm{S} / \mathrm{N}$ for Tier 3 (Tinetti et al. 2018; Edwards et al. 2019a). The retrievals were performed on the scattered spectra and are illustrated in Figure 4. The retrieval maps for the 14 Ariel Tier-3 cases are reported in the Appendix (Figures $\mathrm{C} 1-\mathrm{C} 3$ ) and the molecular detection limits are reported in Table 4. The detection limit for Ariel Tier-3 spectra is very low, and typically mixing ratios equal or smaller than $10^{-7}$ can be retrieved. Even $\mathrm{CO}$ at mixing ratios of $\sim 10^{-6}$ appears to be detectable. Due to the limited number of studied cases, the Tier-3 detection limits reported here should be taken with caution and will be refined in a separate paper dedicated to the study of Tier-3 planets.

\subsection{Biased Sample: Linear Water-temperature Trend}

When we imposed an arbitrary linear trend between the water abundance and the effective temperature, we obtained the watertemperature map shown in Figure 5. Here the imposed trend is easily recovered by our retrieval analysis. Both scattered and unscattered spectra allow one to recover the imposed trend down to water abundances of $\sim 10^{-6}$. In the scattered example, a few cases have larger departures from the true value compared to the nonscattered one but this does not affect the conclusions on the entire population. Additionally, we note that this analysis has been done without retrieval fine tuning.

\subsection{Biased Sample: Equilibrium Chemistry Atmospheres}

When an equilibrium chemistry model was used for both the forward model and the retrievals, we obtained the 

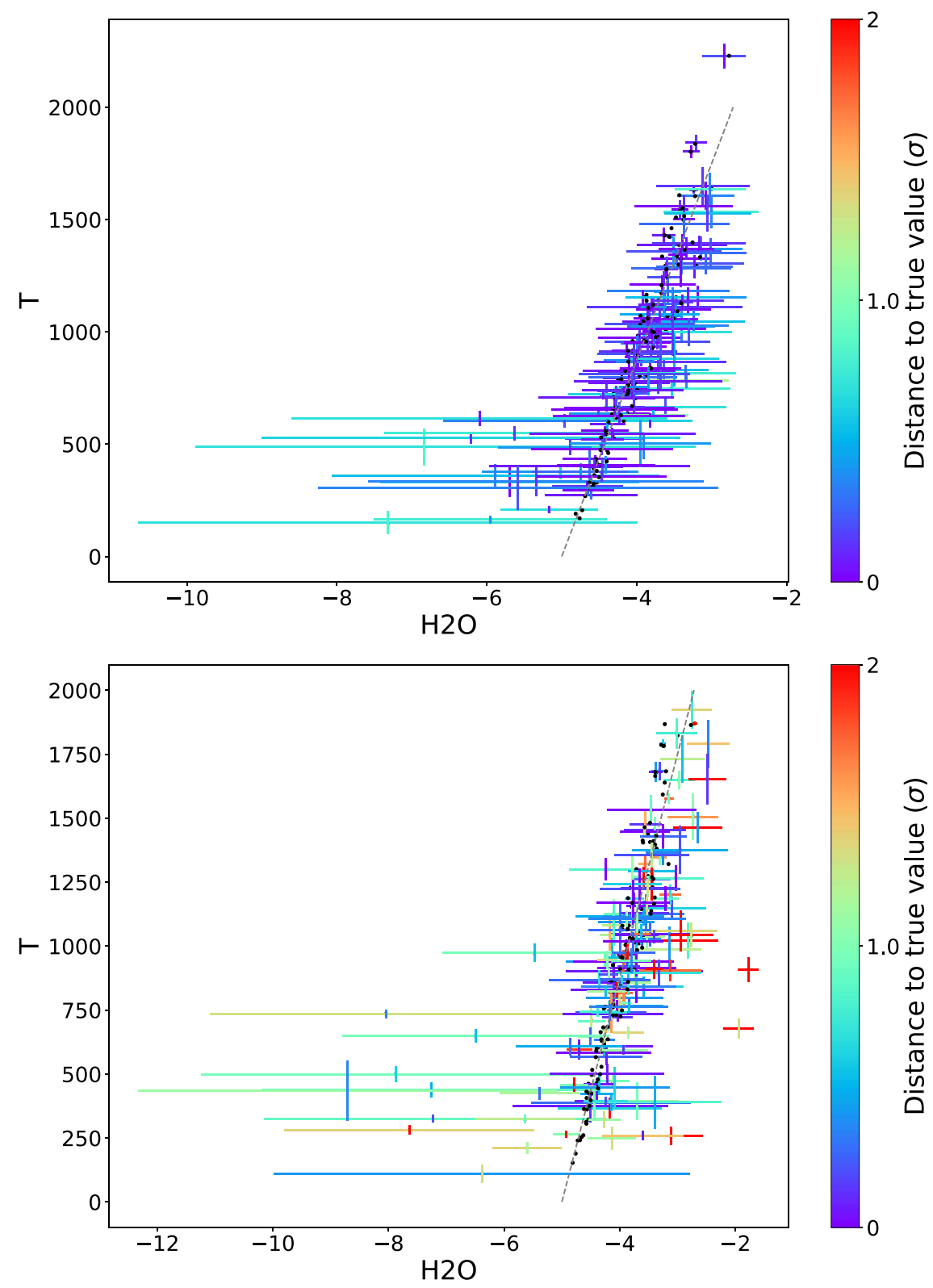

Figure 5. Biased sample: linear water-temperature trend. Top: retrieved water-temperature map from the nonscattered spectra. Bottom: retrieved water-temperature map from the scattered spectra. We show the retrieved $1 \sigma$ error bars on the retrieved parameters. The color scale represents the distance to the true value in units of $1 \sigma$. The dashed gray line indicates the input trend.

water-temperature map shown in Figure 6, top, where the trend is very accurately and precisely recovered. Since the molecular abundances are varying with altitude, the values stated correspond to the average weighted by the atmospheric contribution function (the optical depth variations collapsed over wavelengths). As the models generating and retrieving the data are the same, this is an optimistic result, as we should not expect all atmospheres to satisfy the equilibrium chemistry assumption.

Also the free, constant with pressure chemistry retrievals (Figure 6, bottom) allow one to recover the equilibrium chemistry trend. The retrieved parameters, however, have large distances from the true value, and in some cases the offsets are greater than $2 \sigma$, meaning that the model confidently recovers a biased value. This behavior, also present in other chemical species (see the Appendix, Figures D1-D4), is particularly noticeable for temperatures between 600 and $1100 \mathrm{~K}$ : this region is known to exhibit large chemical gradients with altitude as the balance in the $\mathrm{CH}_{4} / \mathrm{CO}$ reaction changes. These variations in the chemical profiles cannot be captured by our simplistic constant chemistry retrieval model.

It has been shown in Changeat et al. (2019a) that Ariel and JWST will be sensitive to chemical vertical gradients and that retrieval techniques such as the two-layer parameterization would be essential for the analysis of these next-generation spectra.

We show in Figure 7 a comparison between the various retrieval techniques: the two-layer parameterization (Changeat et al. 2019a) well captures the departure of the methane profile from the constant with altitude case without strong prior assumptions, as opposed to the case of the equilibrium chemistry retrieval. 

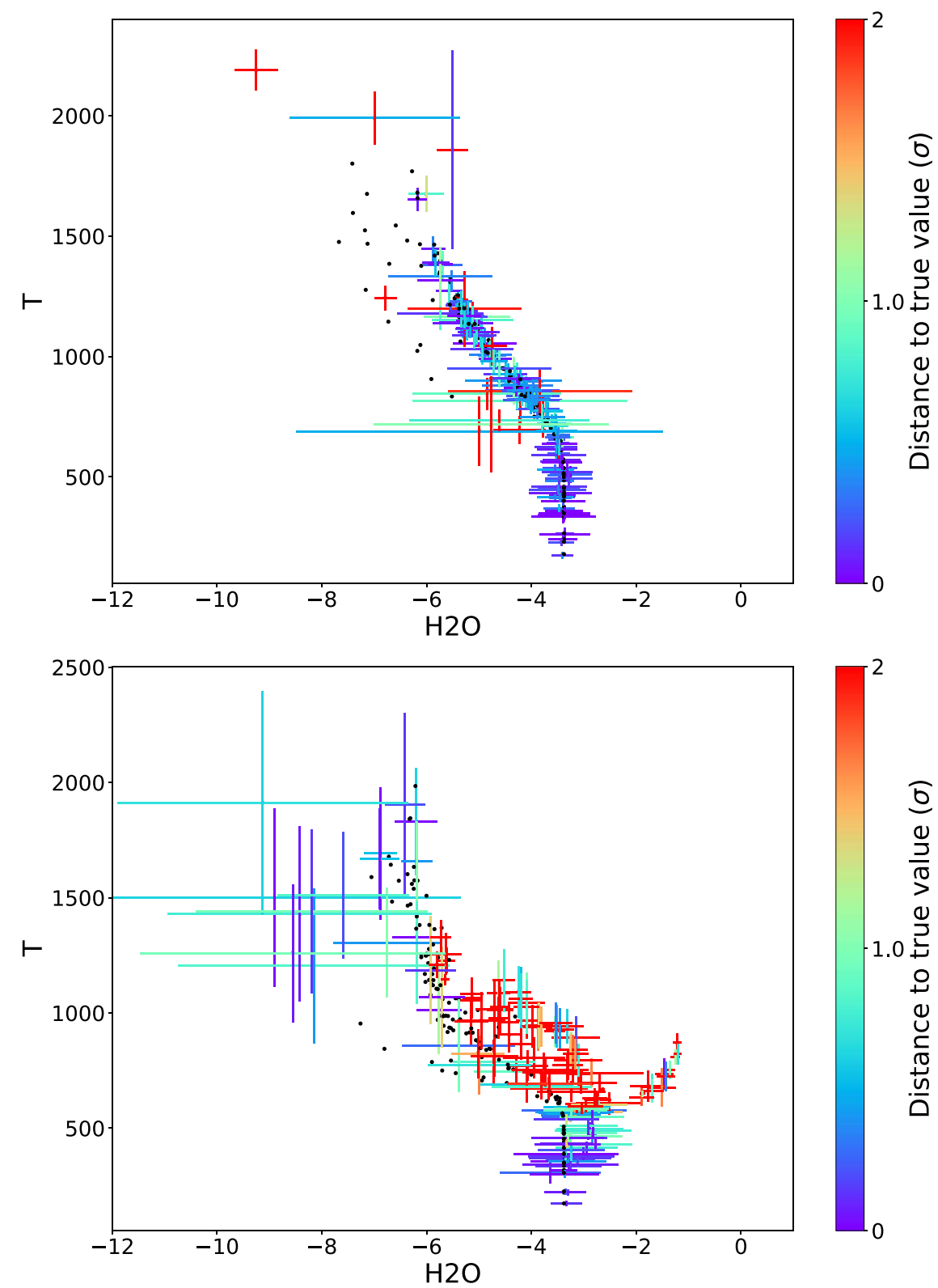

Figure 6. Biased sample: equilibrium chemistry atmospheres. Correlation map of the retrieved abundance of water and the temperature. Results obtained with equilibrium chemistry retrievals (top) and with free, constant with altitude chemistry retrievals (bottom). We show the retrieved $1 \sigma$ error bars on the retrieved parameters. The color scale represents the distance to the true value in units of $1 \sigma$.

\section{Discussion}

In all simulated cases, retrieval analyses were performed without any fine tuning. Also our simulations are simplified compared to real atmospheres, which are expected to have disequilibrium effects, 3D effects, and other complexities.

Recently, self-consistent methods, such as the equilibrium chemistry retrieval adopted in a few examples here, have been implemented in retrieval tools. Embedding these chemical schemes in atmospheric retrievals is very tempting as they allow one to describe complex chemistry while maintaining a low dimensionality. However, we should be careful in using these techniques to interpret unknown atmospheres, as they do not reflect the information content of the observed spectra. In other terms, if the assumptions made by the retrieval model are not correct, the results will likely be biased (Agúndez et al. 2012; Miller-Ricci Kempton et al. 2012; Rocchetto et al. 2016;
Changeat et al. 2019a). This issue has been discussed in the literature and should always be remembered when using such techniques.

Other approaches which let the chemical species assume arbitrary values, may allow for the discovery of unexpected trends in the data. However, the model complexity should be adapted to the data, which is not known a priori. A too simplistic model will tend to be biased, while a too complex model will tend to overfit. In this paper, Section 3.3 highlighted a case where the free constant with pressure chemistry retrieval did not adequately describe the input chemical profiles (which were using equilibrium chemistry), thus biasing our results. A more sophisticated description of the chemical profiles in retrievals is presented in Changeat et al. (2019a).

We illustrate this point by comparing different chemical schemes on an observed spectrum taken from our previously made equilibrium chemistry data set. Figure 7 demonstrates 

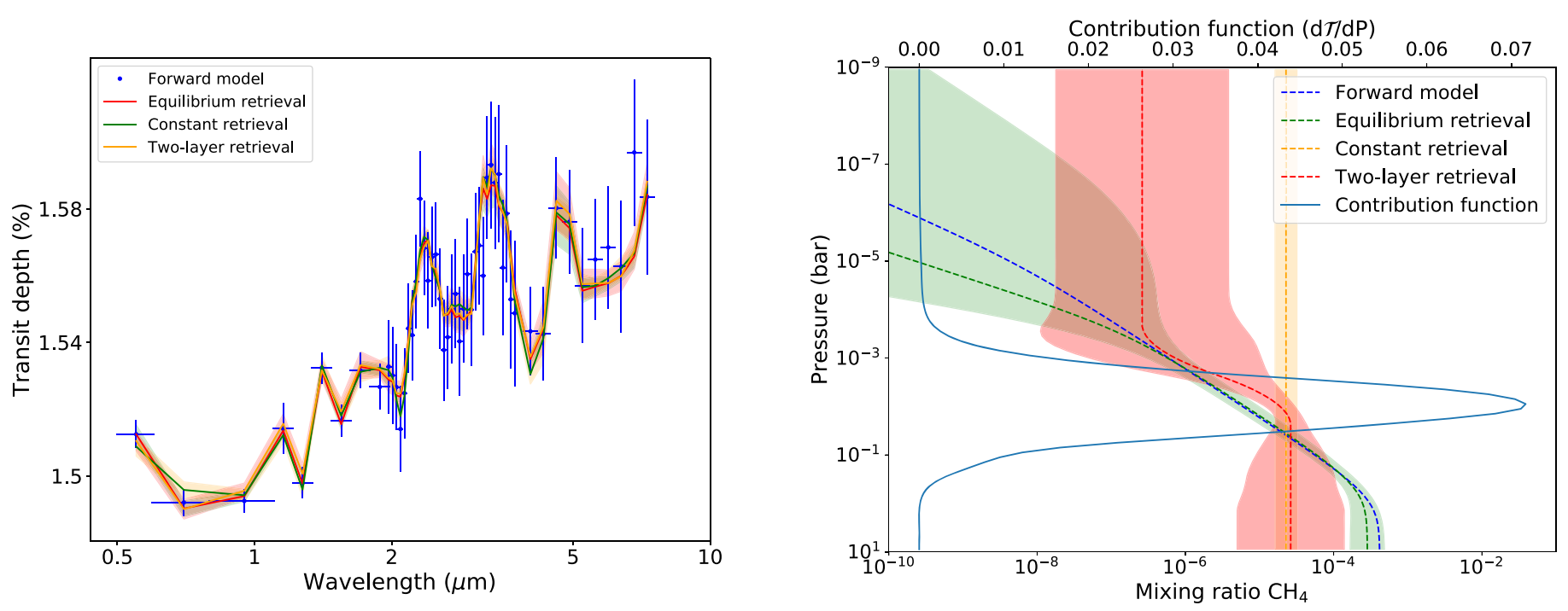

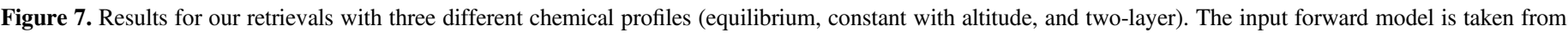

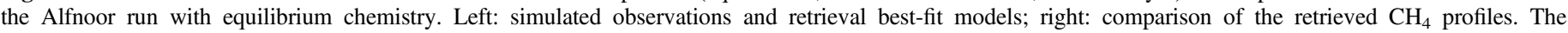

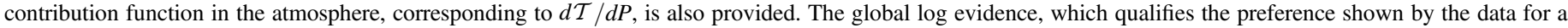

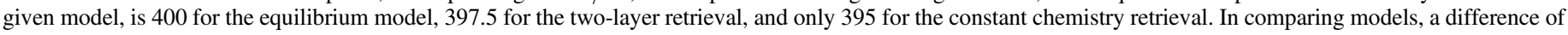

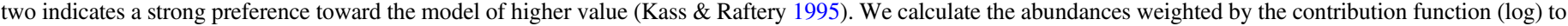

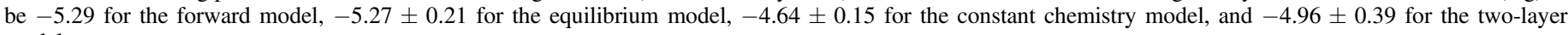
model.

that all three chemical schemes (equilibrium, constant, twolayer) are able to match the observed spectrum. The contribution function (solid blue line on the right figure) shows how the models try to reproduce the input abundances for $\mathrm{CH}_{4}$ in the region where the contribution function is maximum. The equilibrium and two-layer scenarios are better describing the input profiles in general, while the retrieved uncertainties are more representative. The retrieved constant chemical profile with altitude only averages the input $\mathrm{CH}_{4}$ abundance, providing limited details on the atmospheric chemical processes. As expected, we find that the input retrieved weighted abundance is best approximated by the equilibrium model, since this is the same model used to generate the observation (values are stated in Figure 7). The constant with pressure chemistry model is overconfident and is more than $3 \sigma$ offset to the true value. For the two-layer, the true abundance is within the error bars of the retrieved value. The behavior seen in this example explains the large distances to the true value and the general overconfidence in the retrieved chemistry of our free constant with altitude scenario in Figure 6.

\section{Conclusion}

This work assessed the capabilities of Ariel to identify chemical trends - if present-in exoplanet populations through the study of their atmospheres. We developed a dedicated software, Alfnoor, to perform atmospheric retrievals on the entire Ariel list of planetary candidates. Among the key results obtained, we found the detection limits for $\mathrm{H}_{2} \mathrm{O}, \mathrm{CH}_{4}, \mathrm{CO}_{2}$, and $\mathrm{NH}_{3}$ to be $\sim 10^{-6}$ in the case of Tier 2 and $<10^{-7}$ in the case of Tier- 3 transit observations. $\mathrm{CO}$, though, has higher detection thresholds, i.e., $\sim 10^{-4}$ for Tier- 2 observations and $\sim 10^{-6}$ for Tier 3 .

We also confirmed the potential of Ariel to recover chemical trends in exoplanetary atmospheres. We tested correlations between chemical species and temperature and a planet population whose chemical composition is entirely determined by equilibrium chemistry.

Limitations in our assumptions for the chemistry, temperature, and cloud models imply that additional work still needs to be done to fully understand the degeneracies associated with these techniques and how to fully automate retrieval strategies. In the future, we aim to simulate more realistic scenarios using self-consistent forward models (e.g., including disequilibrium chemistry) and more complex thermal and cloud assumptions. While this work was inspired by the Ariel mission, similar large-scale simulations could also help prioritizing the use of other observatories from space and the ground and provide a great tool for the preparation of observational campaigns.

This project has received funding from the European Research Council (ERC) under the European Union's Horizon 2020 research and innovation program (grant agreement No. 758892, ExoAI) and under the European Union's Seventh Framework Programme (FP7/2007-2013)/ERC grant agreement numbers 617119 (ExoLights). Furthermore, we acknowledge funding by the Science and Technology Funding Council (STFC) grants: ST/K502406/1, ST/P000282/1, ST/P002153/1, and ST/S002634/1 ASI grant no. 2018.22.HH.O. UCL LondonRome Cities Partnerships Program.

\section{Appendix A \\ Complementary Figures to the Unbiased Sample}

The complementary chemistry-temperature maps $\left(\mathrm{CH}_{4}, \mathrm{CO}\right.$, $\mathrm{CO}_{2}$, and $\mathrm{NH}_{3}$ ) to the Alfnoor unbiased sample are shown in Figures A1-A4. Figure A5 presents the retrieved radius versus cloud pressure map. We also provide the complementary true versus retrieved plots and error retrieved for each molecule in Figures A6-A9. 

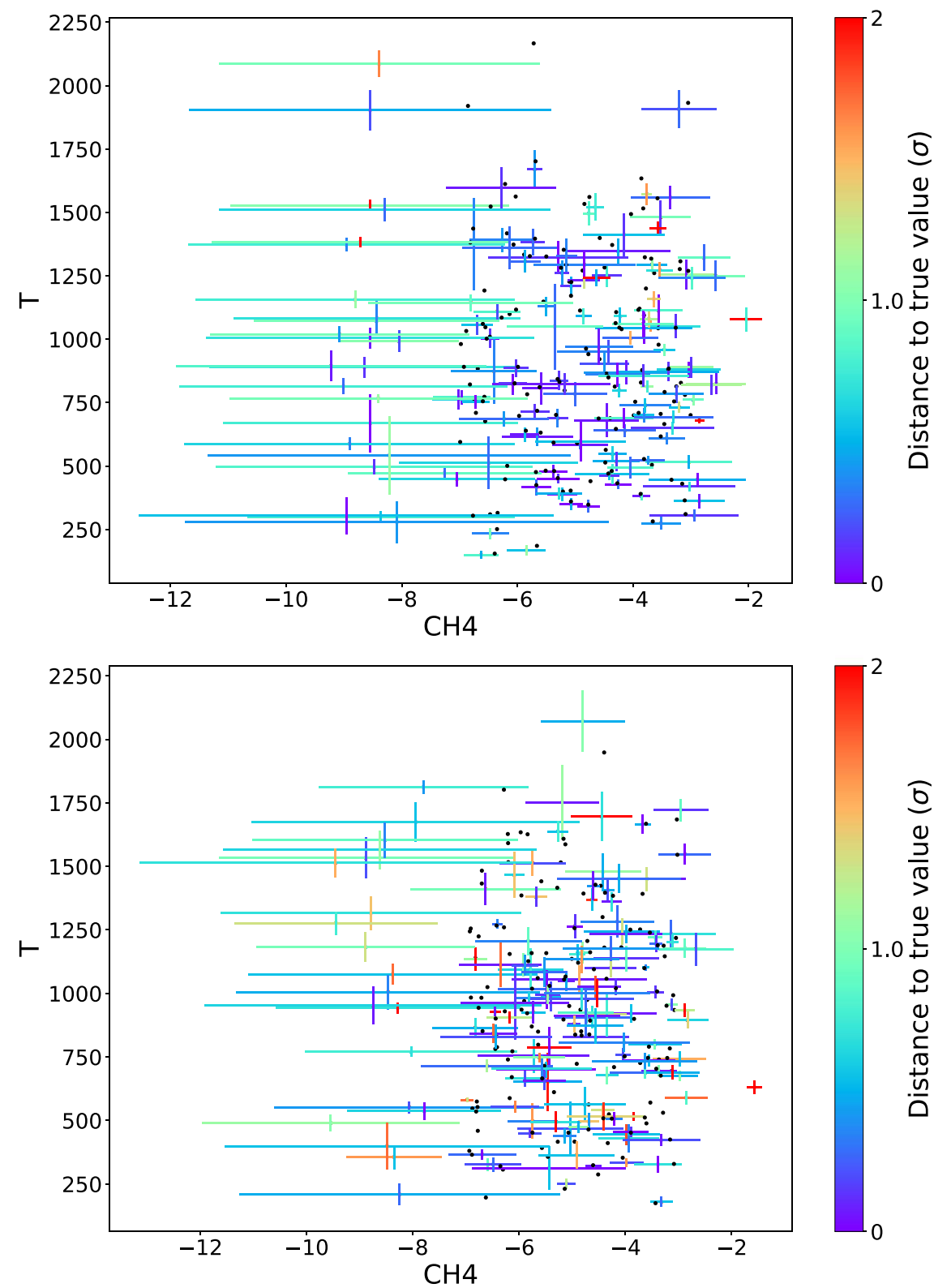

Figure A1. Unbiased sample: correlation map between the temperature and the retrieved abundances of $\mathrm{CH}_{4}$, with the $1 \sigma$ retrieved error bars. The color scale represents the distance to the true value in units of $1 \sigma$. Top: nonscattered spectra. Bottom: scattered spectra. 

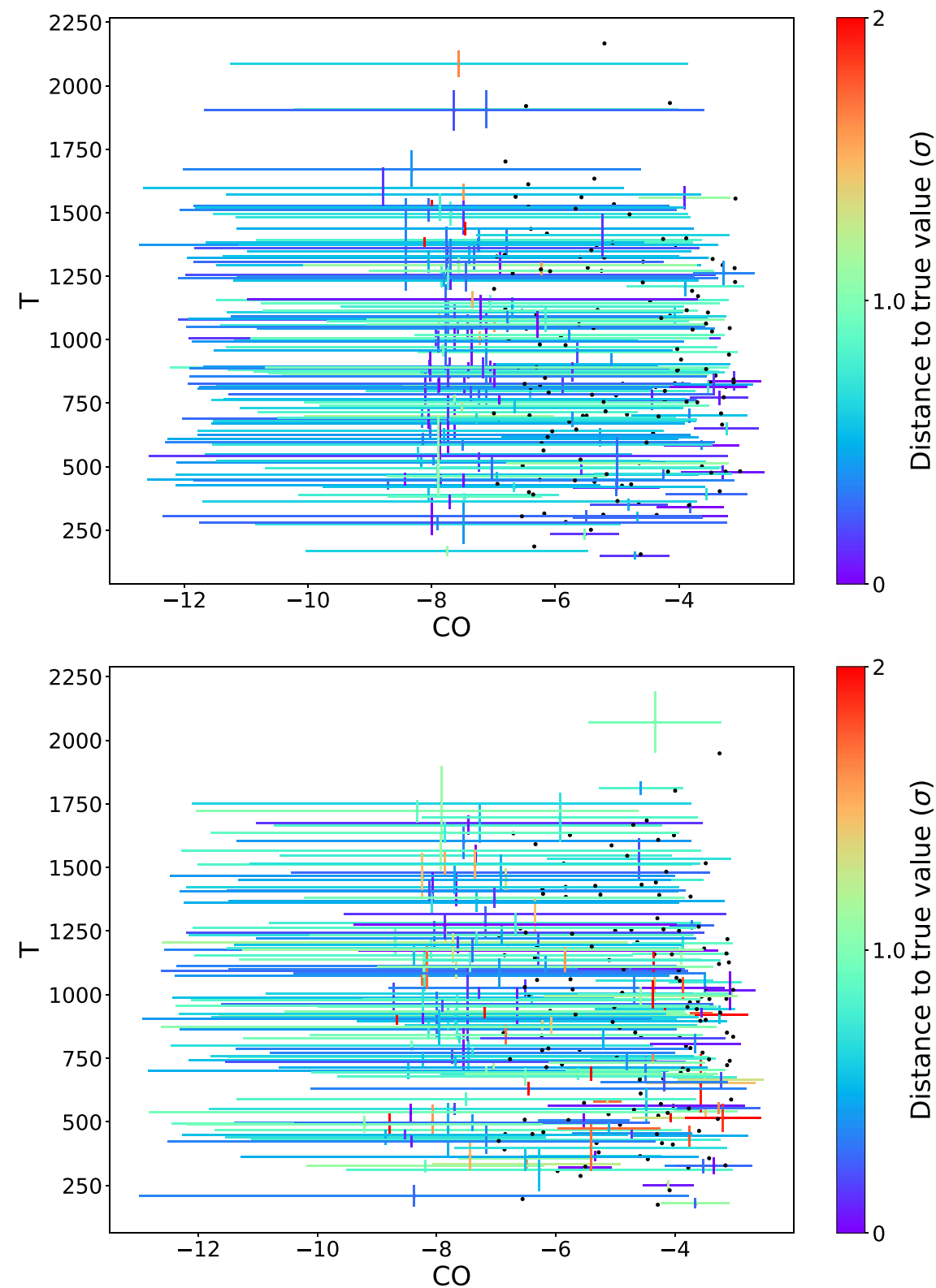

Figure A2. Unbiased sample: correlation map between the temperature and the retrieved abundances of CO, with the $1 \sigma$ retrieved error bars. The color scale represents the distance to the true value in units of $1 \sigma$. Top: nonscattered spectra. Bottom: scattered spectra. 

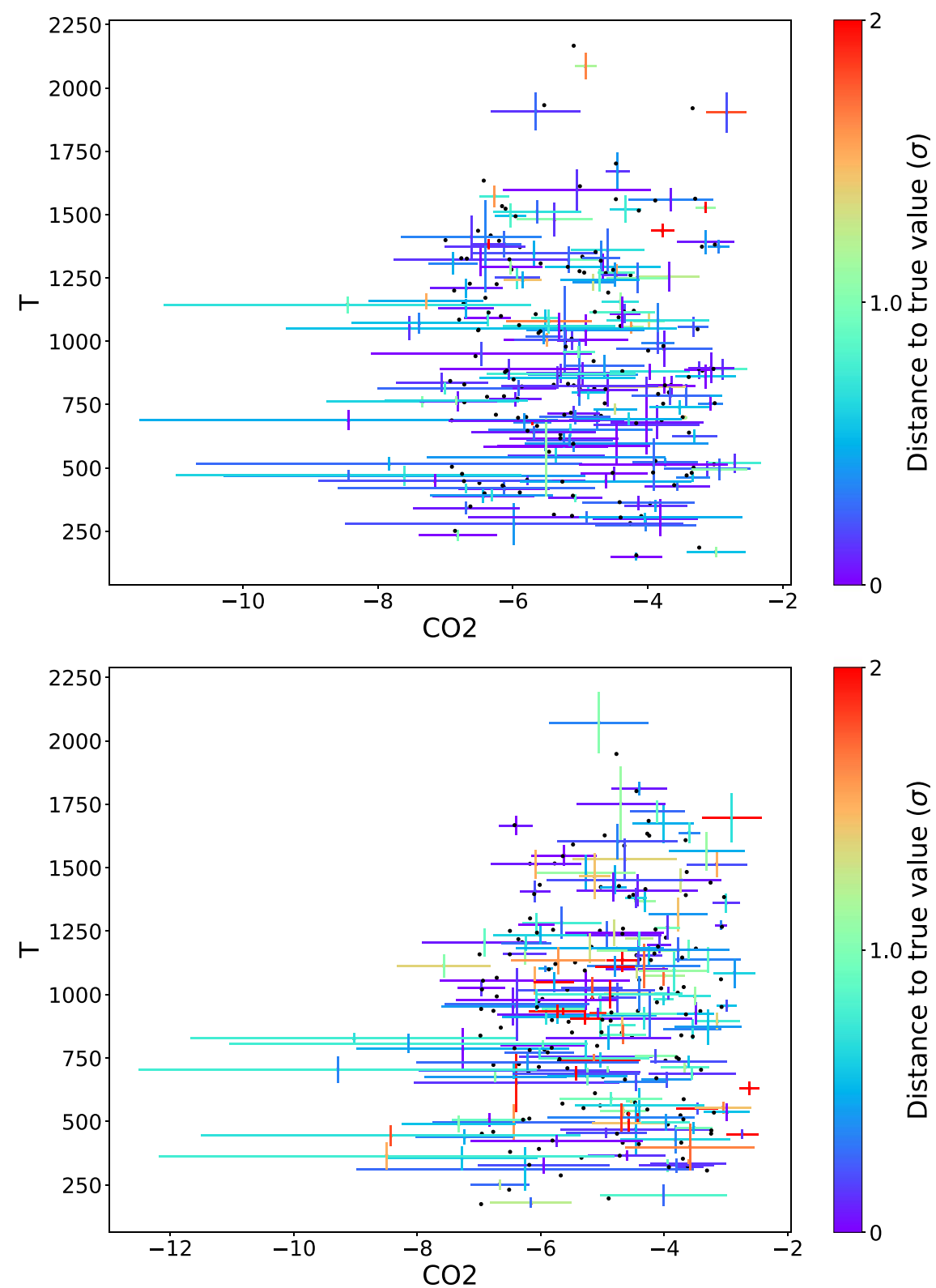

Figure A3. Unbiased sample: correlation map between the temperature and the retrieved abundances of $\mathrm{CO}_{2}$, with the $1 \sigma$ retrieved error bars. The color scale represents the distance to the true value in units of $1 \sigma$. Top: nonscattered spectra. Bottom: scattered spectra. 

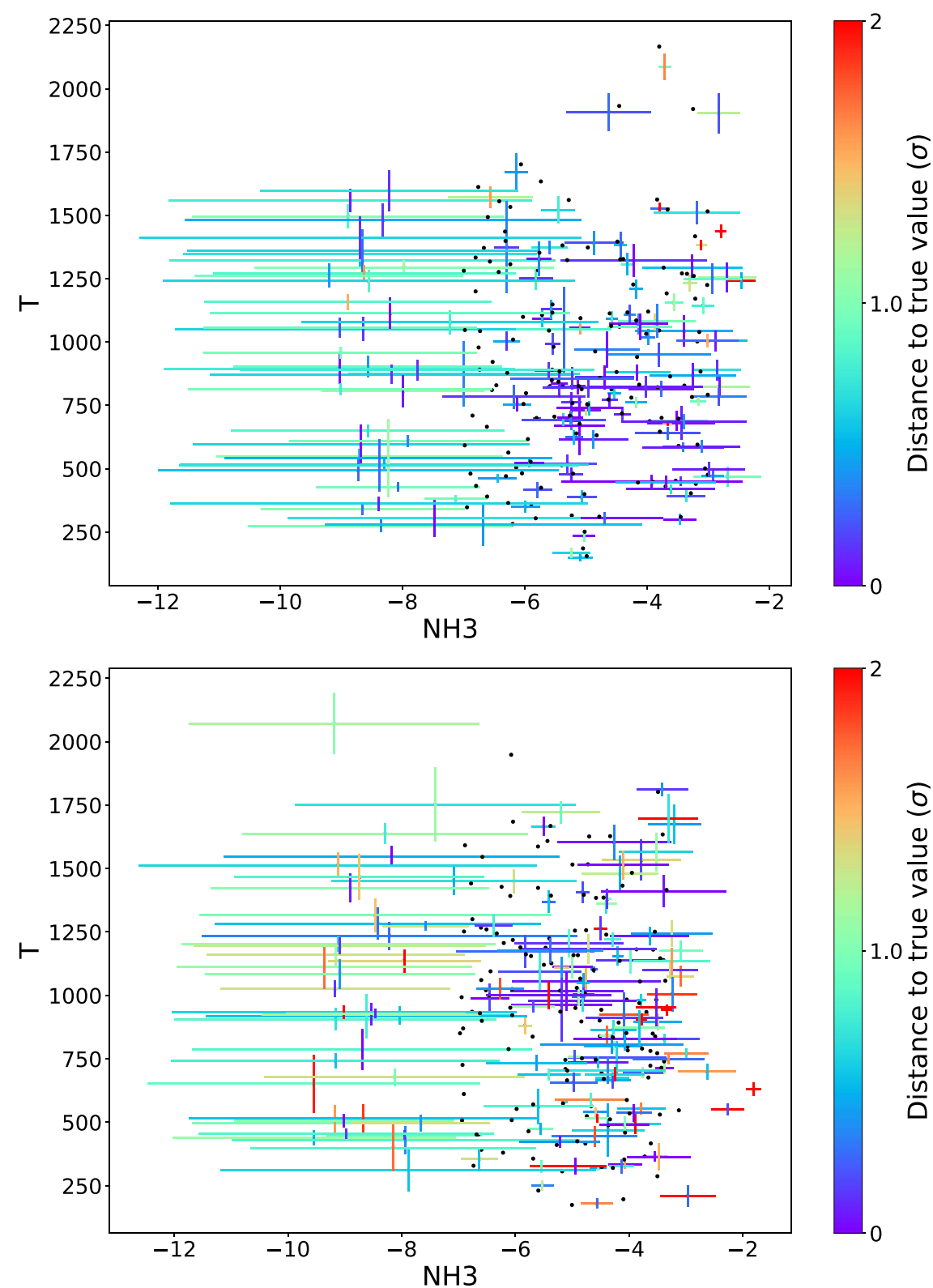

Figure A4. Unbiased sample: correlation map between the temperature and the retrieved abundances of $\mathrm{NH}_{3}$, with the $1 \sigma$ retrieved error bars. The color scale represents the distance to the true value in units of $1 \sigma$. Top: nonscattered spectra. Bottom: scattered spectra. 

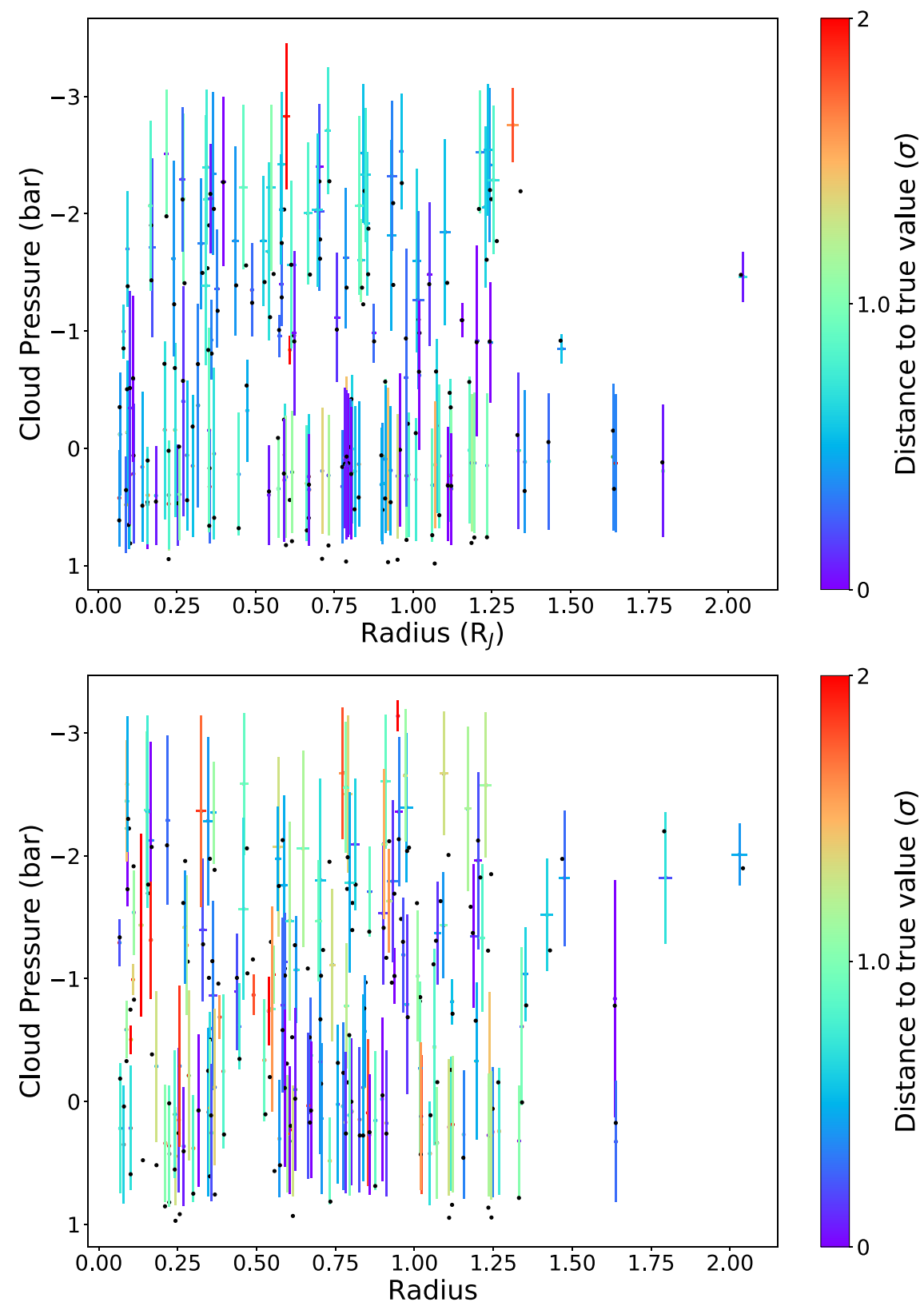

Figure A5. Unbiased sample: correlation map between the clouds and the radius, with the $1 \sigma$ retrieved error bars. The color scale represents the distance to the true value in units of $1 \sigma$. Top: nonscattered spectra. Bottom: scattered spectra. 

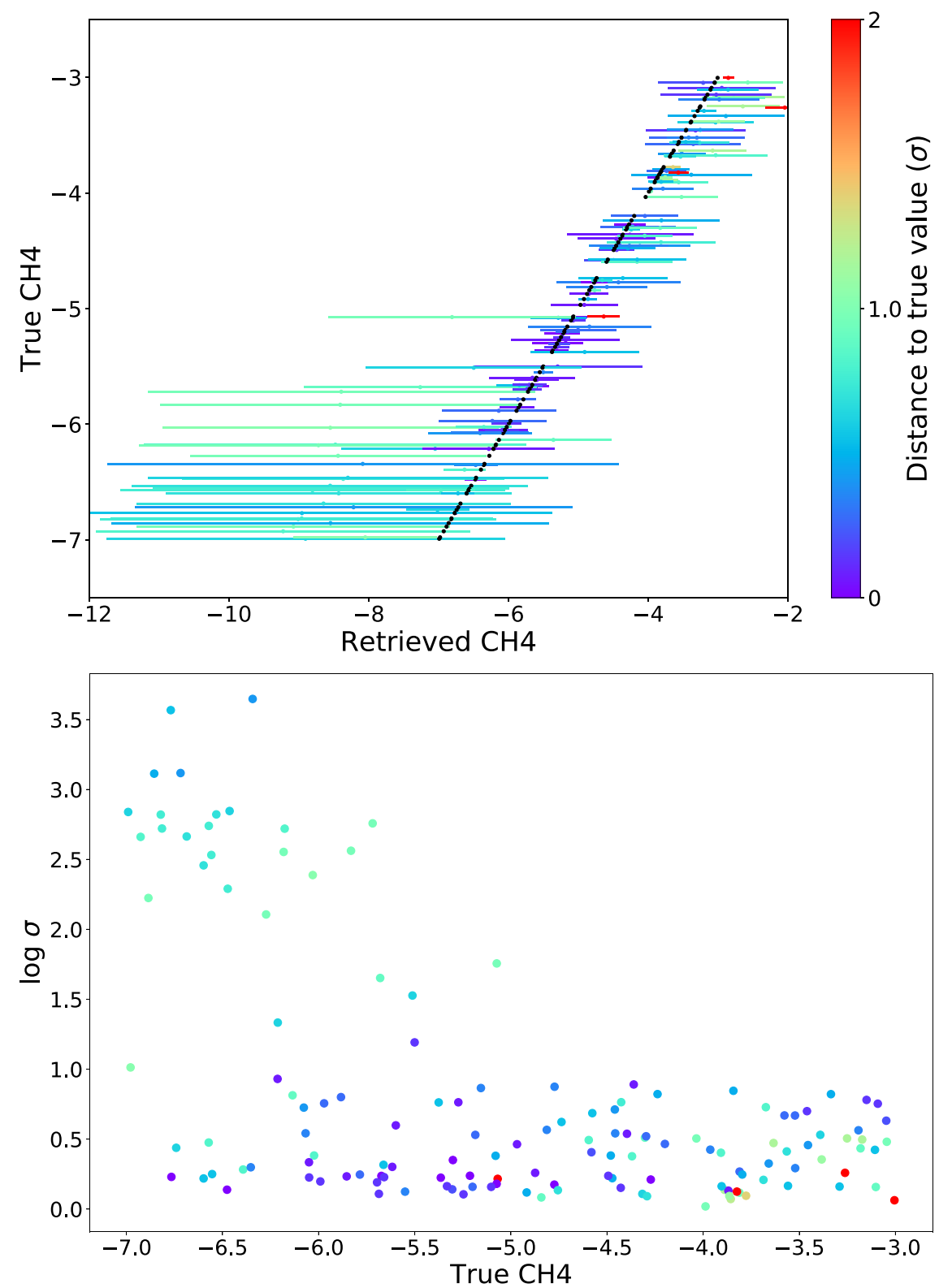

Figure A6. Top: map of the $\mathrm{CH}_{4}$ retrieved abundances vs. their values for the unbiased sample. Bottom: error retrieved as a function of the input abundances. The color scale of the $1 \sigma$ retrieved error bars represents the distance to the true value in units of $1 \sigma$. 

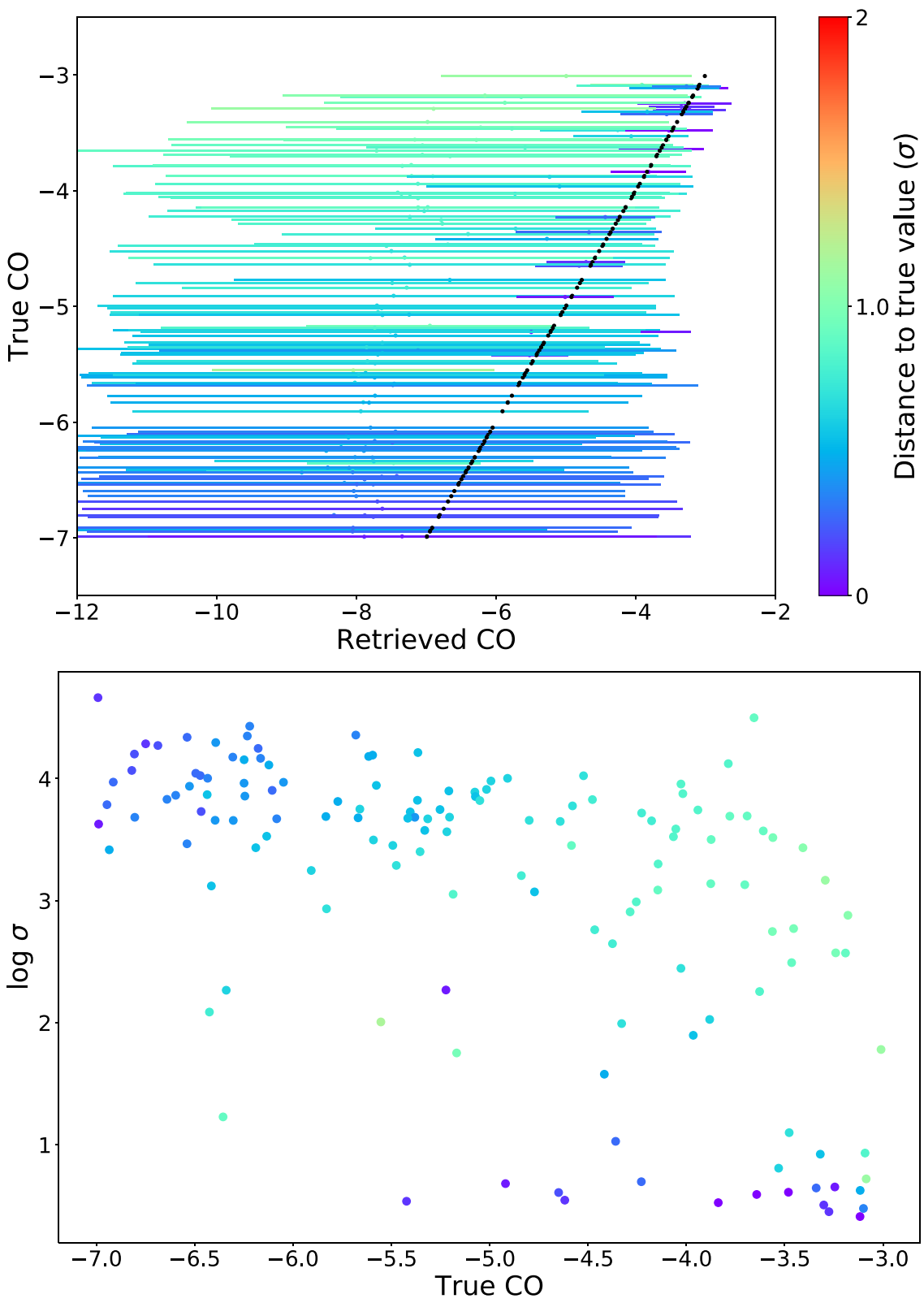

Figure A7. Top: map of the CO retrieved abundances vs. their values for the unbiased sample. Bottom: Error retrieved as a function of the input abundances. The color scale of the $1 \sigma$ retrieved error bars represents the distance to the true value in units of $1 \sigma$. 

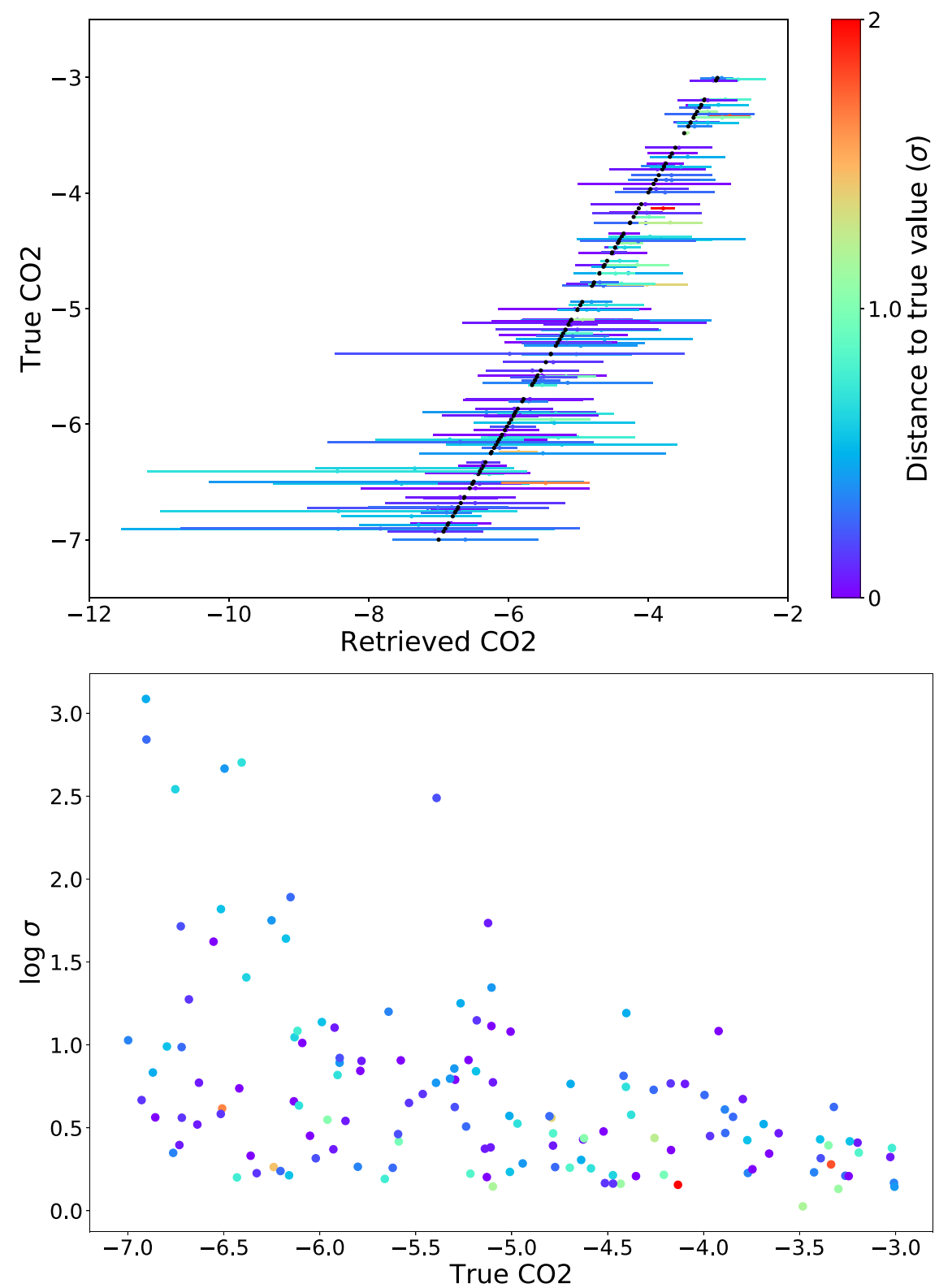

Figure A8. Top: map of the $\mathrm{CO}_{2}$ retrieved abundances vs. their values for the unbiased sample. Bottom: error retrieved as a function of the input abundances. The color scale of the $1 \sigma$ retrieved error bars represents the distance to the true value in units of $1 \sigma$. 

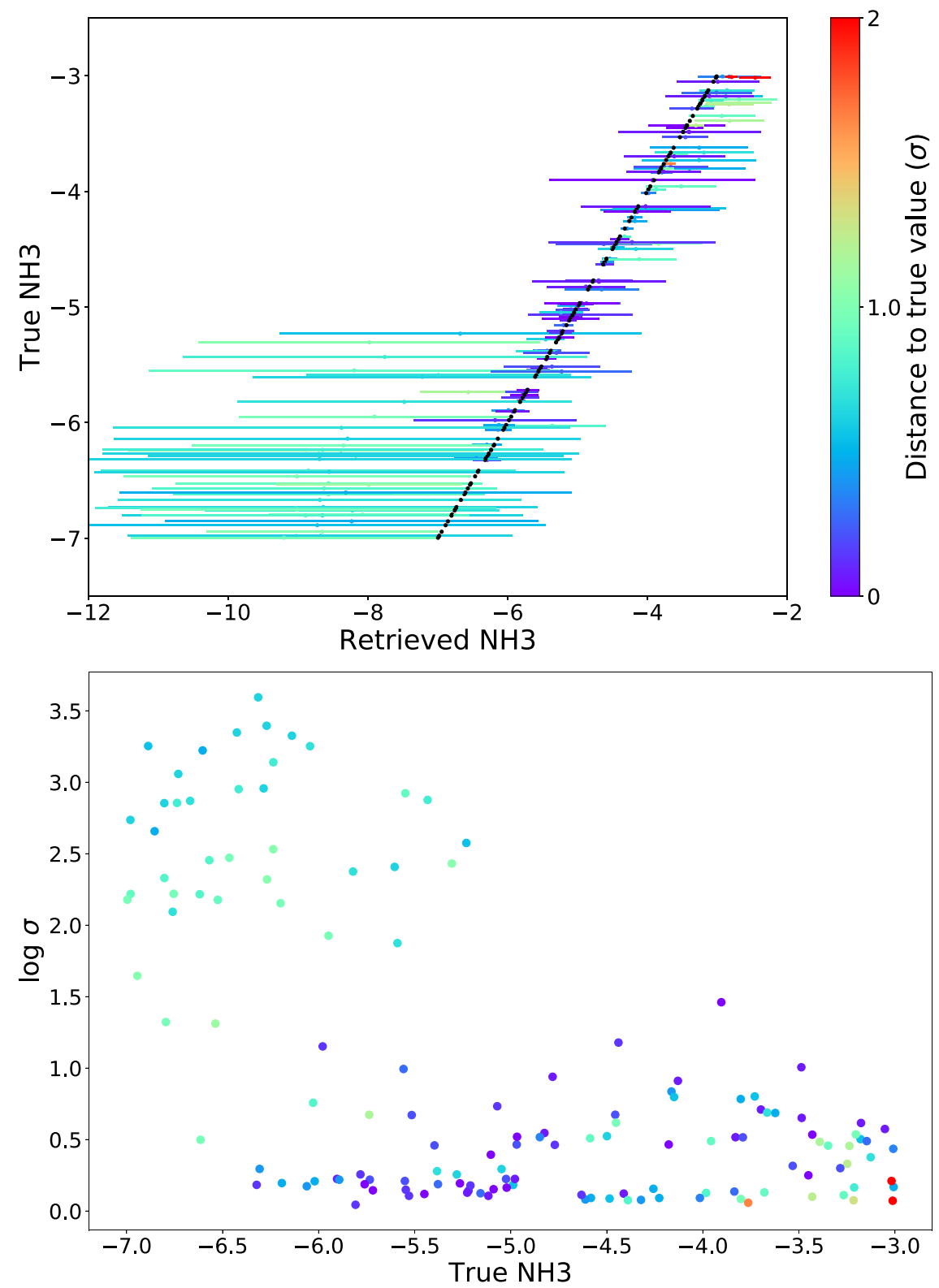

Figure A9. Top: map of the $\mathrm{NH}_{3}$ retrieved abundances vs. their values for the unbiased sample. Bottom: error retrieved as a function of the input abundances. The color scale of the $1 \sigma$ retrieved error bars represents the distance to the true value in units of $1 \sigma$. 


\section{Appendix B}

\section{Absorption Contributions in Ariel Spectra of the Molecules}

\section{Considered in This Work}

Figure $\mathrm{B} 1$ presents the absorption of different species $\left(\mathrm{H}_{2} \mathrm{O}\right.$,

$\mathrm{CO}, \mathrm{CO}_{2}$, and $\mathrm{NH}_{3}$ ) in Ariel observations.

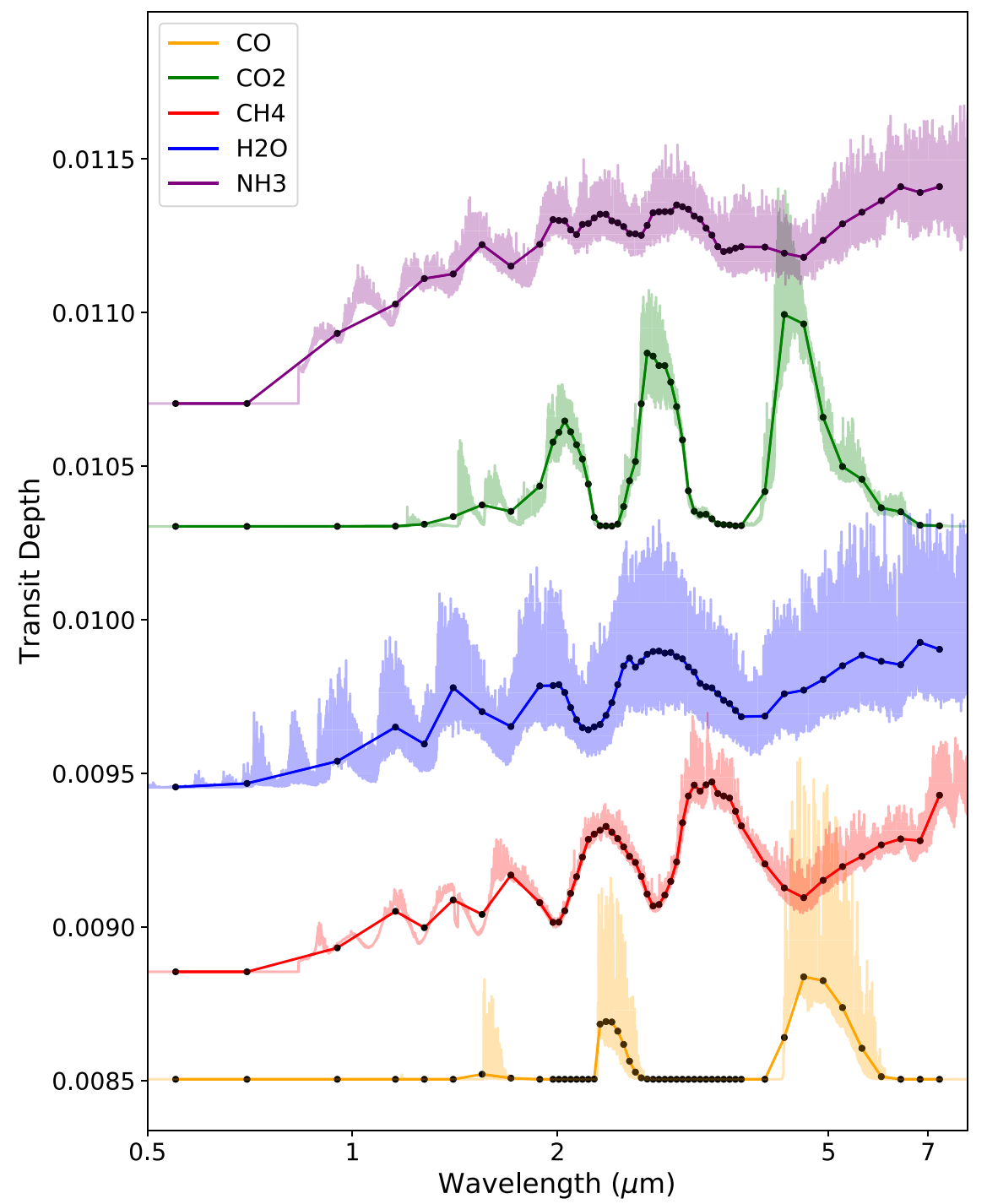

Figure B1. Absorption in Ariel of the molecular species considered in this paper. Each simulation is for a $1 R_{\mathrm{J}}, 1 M_{\mathrm{J}}$ planet and $1 R_{\mathrm{S}}$ star with $10^{-5}$ of the considered molecule as only absorber. The models are offset for better visibility. The shaded region is the full resolution contribution, while the solid lines and black points correspond to Ariel resolutions. 


\section{Appendix C}

\section{Complementary Figures to the Unbiased Run Using Ariel}

\section{Tier 3 Noise}

The complementary temperature-chemistry maps to our Ariel Tier 3 unbiased run are shown in Figures C1-C3.
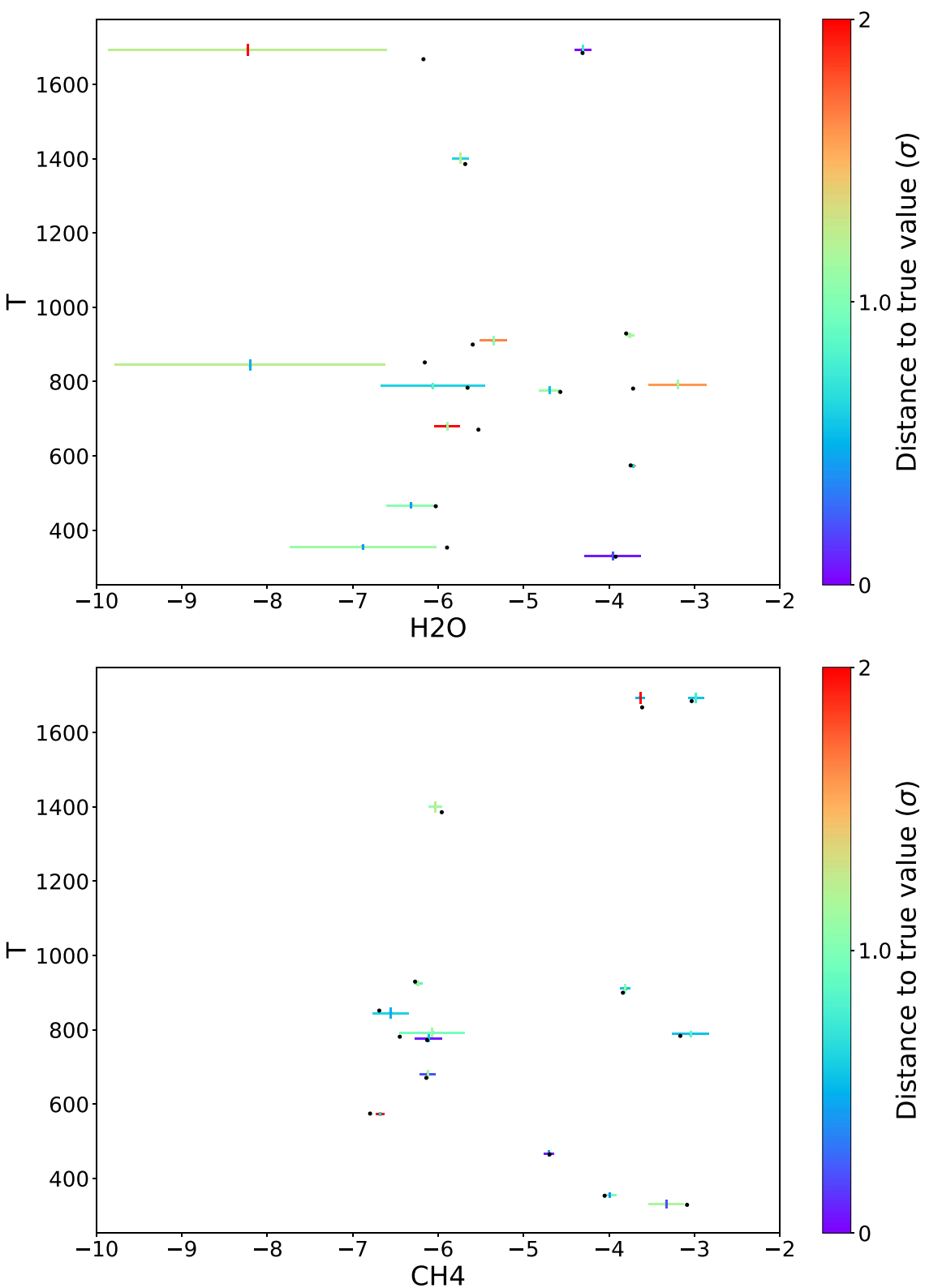

Figure C1. Correlations maps obtained for Ariel Tier-3 scattered spectra: $\mathrm{H}_{2} \mathrm{O}-T$ (top) and $\mathrm{CH}_{4}-T$ (bottom). 

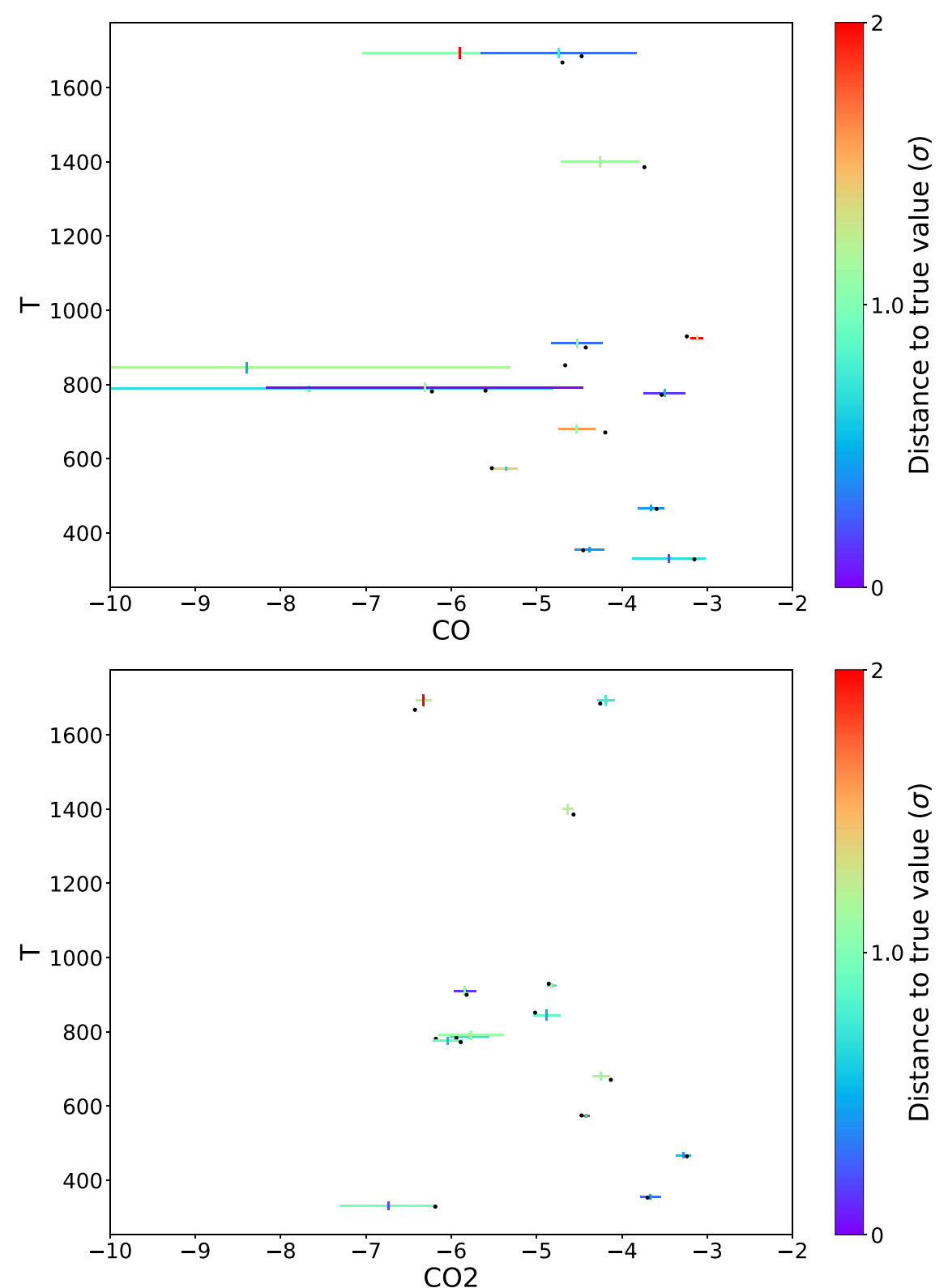

Figure C2. Correlations maps obtained for Ariel Tier-3 scattered spectra: $\mathrm{CO}-T$ (top) and $\mathrm{CO}_{2}-T$ (bottom).

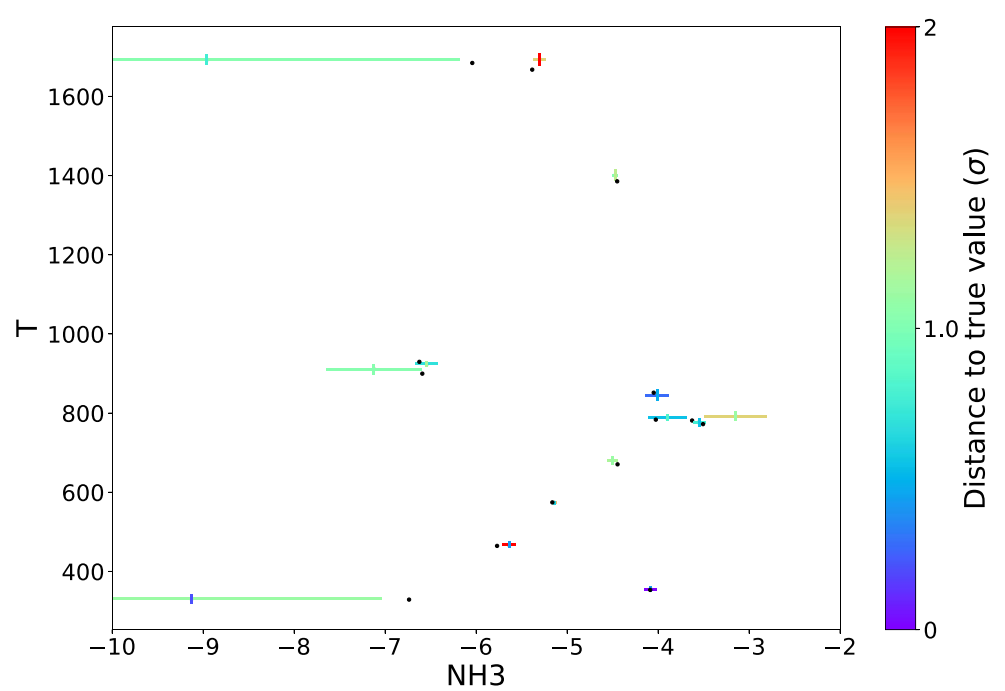

Figure C3. Correlations maps obtained for Ariel Tier-3 scattered spectra: $\mathrm{NH}_{3}-T$. 


\section{Appendix D \\ Complementary Figures to Our Equilibrium Chemistry Runs}

Figures D1-D4 show the complementary temperaturechemistry maps for the Alfnoor runs simulating planets in equilibrium chemistry. Each figure show both equilibrium chemistry retrievals and free chemistry retrievals.
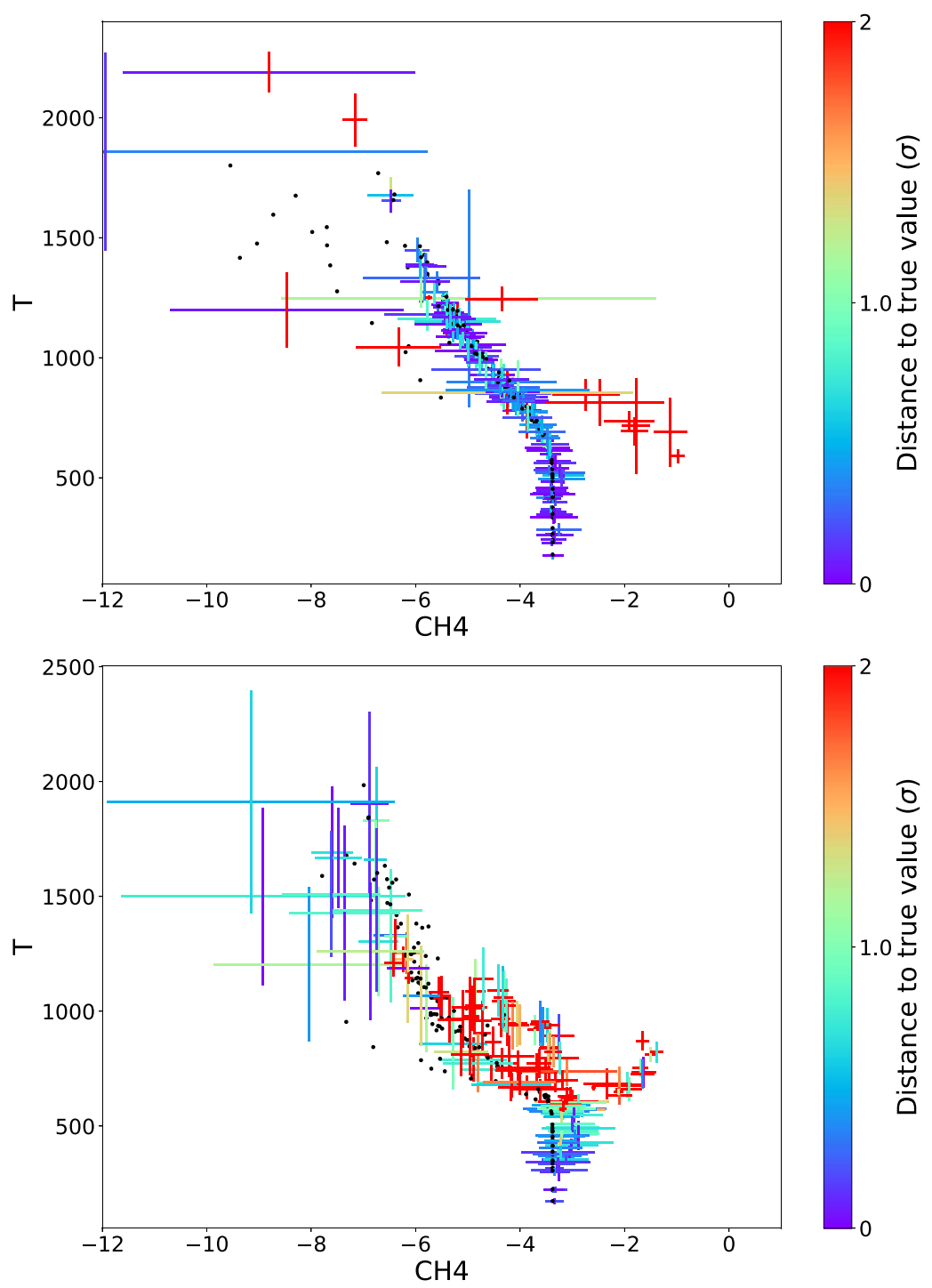

Figure D1. Biased sample: equilibrium chemistry atmospheres. Correlation map of the retrieved abundance of $\mathrm{CH}_{4}$ and the temperature, with the $1 \sigma$ retrieved error bars. Results obtained with equilibrium chemistry retrievals (top) and with free, constant chemistry with pressure retrievals (bottom). The color scale represents the distance to the true value in units of $1 \sigma$. 

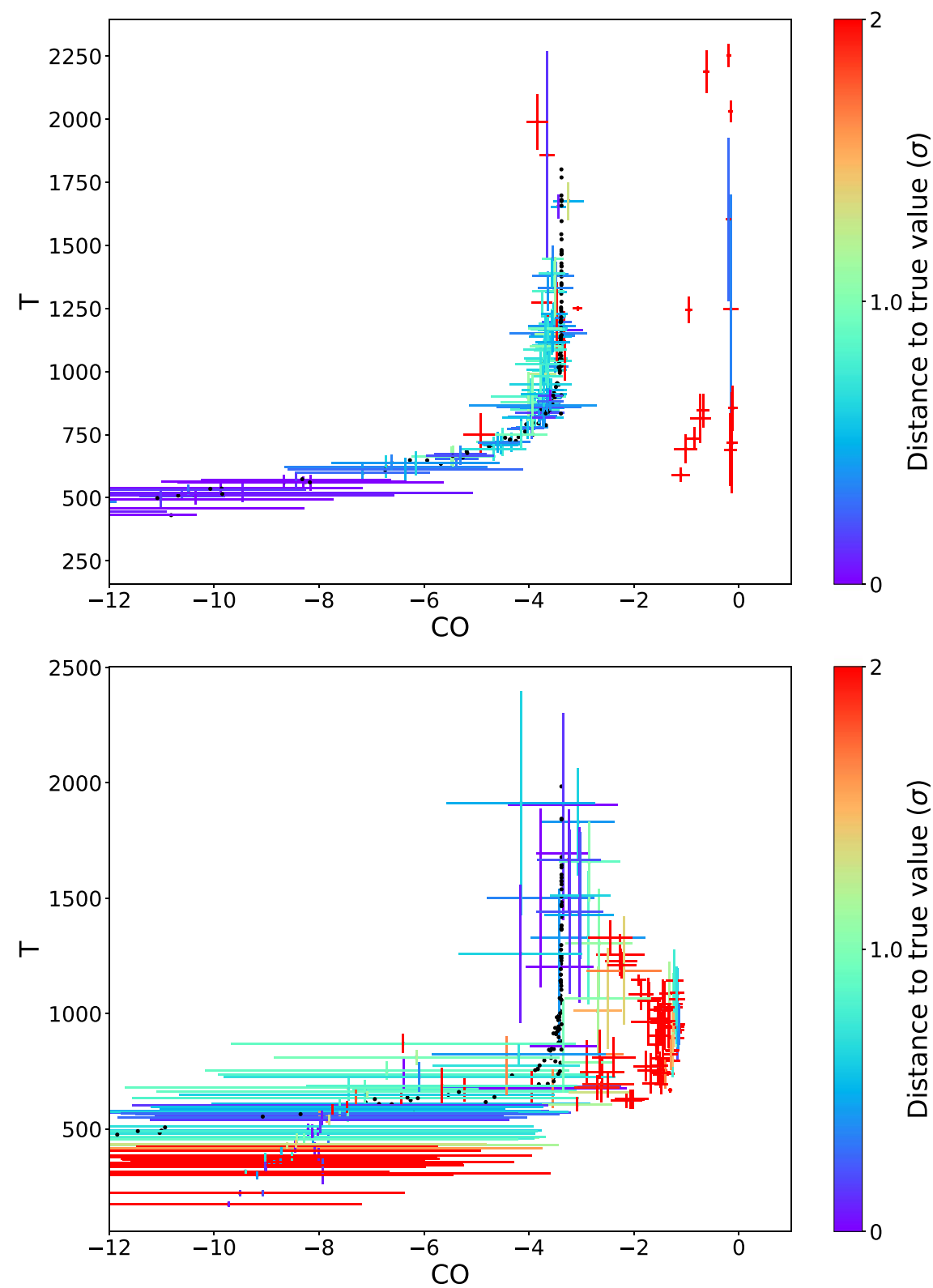

Figure D2. Biased sample: equilibrium chemistry atmospheres. Correlation map of the retrieved abundance of $\mathrm{CO}$ and the temperature, with the $1 \sigma$ retrieved error bars. Results obtained with equilibrium chemistry retrievals (top) and with free, constant with pressure chemistry retrievals (bottom). The color scale represents the distance to the true value in units of $1 \sigma$. 

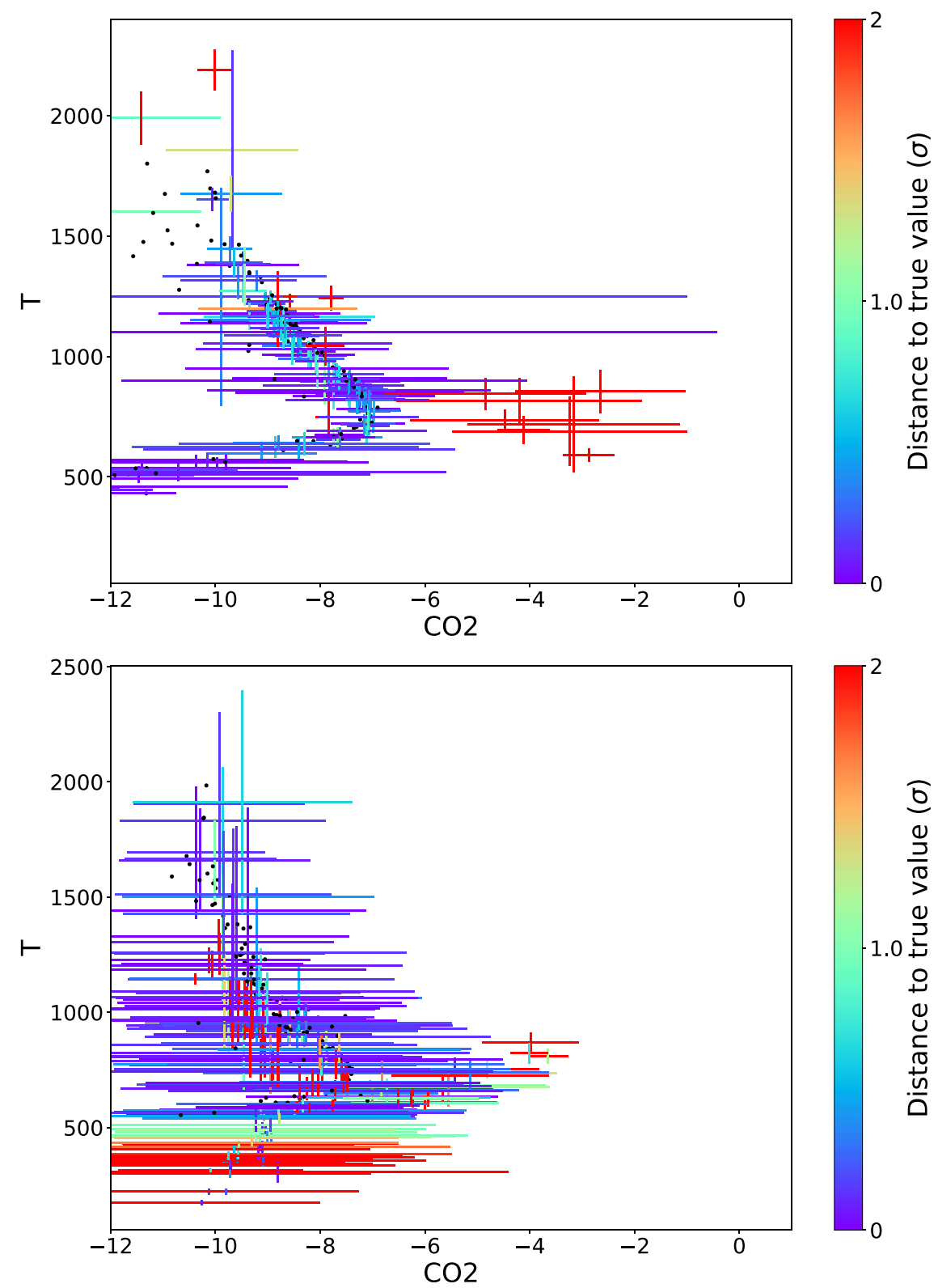

Figure D3. Biased sample: equilibrium chemistry atmospheres. Correlation map of the retrieved abundance of $\mathrm{CO}_{2}$ and the temperature, with the $1 \sigma$ retrieved error bars. Results obtained with equilibrium chemistry retrievals (top) and with free, constant with pressure chemistry retrievals (bottom). The color scale represents the distance to the true value in units of $1 \sigma$ 

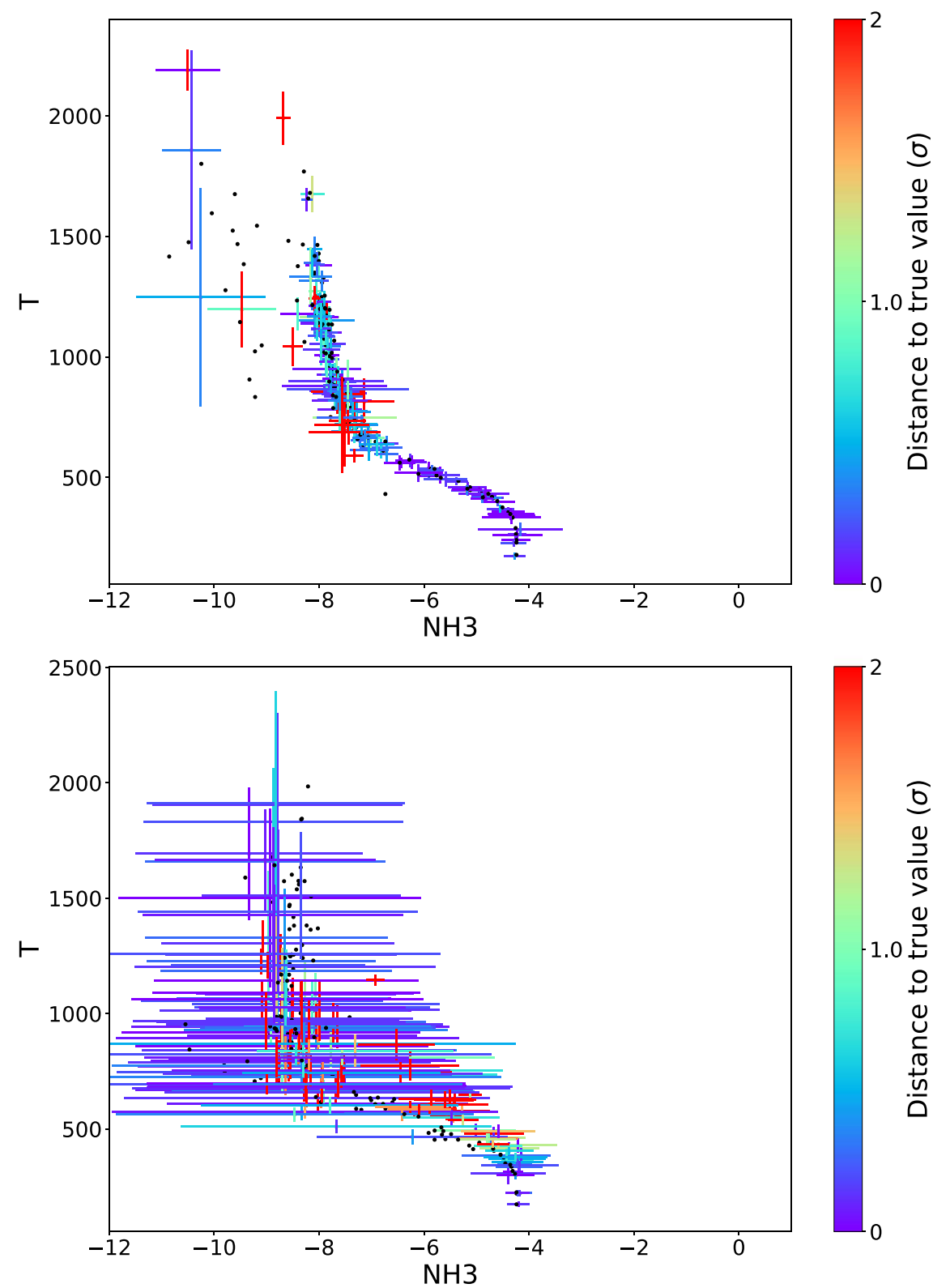

Figure D4. Biased sample: equilibrium chemistry atmospheres. Correlation map of the retrieved abundance of $\mathrm{NH}_{3}$ and the temperature, with the $1 \sigma$ retrieved error bars. Results obtained with equilibrium chemistry retrievals (top) and with free, constant with pressure chemistry retrievals (bottom). The color scale represents the distance to the true value in units of $1 \sigma$.

\section{ORCID iDs}

Q. Changeat (1) https://orcid.org/0000-0001-6516-4493

A. Al-Refaie (ii) https://orcid.org/0000-0003-2241-5330

L. V. Mugnai (1) https://orcid.org/0000-0002-9007-9802

B. Edwards (1) https://orcid.org/0000-0002-5494-3237

I. P. Waldmann (10) https://orcid.org/0000-0002-4205-5267

G. Tinetti (i) https://orcid.org/0000-0001-6058-6654

\section{References}

Abel, M., Frommhold, L., Li, X., \& Hunt, K. L. 2011, JPCA, 115, 6805 Abel, M., Frommhold, L., Li, X., \& Hunt, K. L. 2012, JChPh, 136, 044319 Agúndez, M., Venot, O., Iro, N., et al. 2012, A\&A, 548, A73

Al-Refaie, A. F., Changeat, Q., Waldmann, I. P., \& Tinetti, G. 2019 , arXiv: 1912.07759

Barstow, J. K., Aigrain, S., Irwin, P. G. J., et al. 2012, arXiv:1212.5020

Barstow, J. K., Aigrain, S., Irwin, P. G. J., \& Sing, D. K. 2016, ApJ, 834, 50

Barton, E. J., Hill, C., Yurchenko, S. N., et al. 2017, JQSRT, 187, 453
Batalha, N. E., Mandell, A., Pontoppidan, K., et al. 2017, PASP, 129, 064501 Bennett, D. P., Akeson, R., Anderson, J., et al. 2018, arXiv:1803.08564 Beuzit, J. L., Vigan, A., Mouillet, D., et al. 2019, A\&A, 631, A155 Blumenthal, S. D., Mandell, A. M., Hébrard, E., et al. 2018, ApJ, 853, 138 Bohren, C. F., \& Huffman, D. R. 2008, Absorption and Scattering of Light by Small Particles (Weinheim: John Wiley \& Sons)

Broeg, C., Fortier, A., Ehrenreich, D., et al. 2013, EPJWC, 47, 03005

Caldas, A., Leconte, J., Selsis, F., et al. 2019, A\&A, 623, A161

Cameron, A. C., Guenther, E., Smalley, B., et al. 2010, MNRAS, 407, 507

Changeat, Q., Edwards, B., Waldmann, I. P., \& Tinetti, G. 2019a, ApJ, 886, 39 Changeat, Q., Keyte, L., Waldmann, I. P., \& Tinetti, G. 2019b, ApJ, 896, 107 Delrez, L., Santerne, A., Almenara, J.-M., et al. 2016, MNRAS, 458, 4025 Edwards, B., Mugnai, L., Tinetti, G., Pascale, E., \& Sarkar, S. 2019a, AJ, 157,242

Edwards, B., Rice, M., Zingales, T., et al. 2019b, ExA, 47, 29

Feng, Y. K., Line, M. R., Fortney, J. J., et al. 2016, ApJ, 829, 52

Feng, Y. K., Robinson, T. D., Fortney, J. J., et al. 2018, AJ, 155, 200

Fletcher, L. N., Gustafsson, M., \& Orton, G. S. 2018, ApJS, 235, 24

Gaia Collaboration, Prusti, T., de Bruijne, J. H. J., et al. 2016, A\&A, 595, A1

Gardner, J. P., Mather, J. C., Clampin, M., et al. 2006, SSRv, 123, 485

Gaudi, B. S., Stassun, K. G., Collins, K. A., et al. 2017, Natur, 546, 514 
Gillon, M., Jehin, E., Lederer, S. M., et al. 2016, Natur, 533, 221

Gilmozzi, R., \& Spyromilio, J. 2007, Msngr, 127, 11

Gordon, I., Rothman, L. S., Wilzewski, J. S., et al. 2016, AAS/DPS Meeting, $48,421.13$

Guillot, T. 2010, A\&A, 520, A27

Hill, C., Yurchenko, S. N., \& Tennyson, J. 2013, Icar, 226, 1673

Kass, R. E., \& Raftery, A. E. 1995, Journal of the American Statistical Association, 90,773

Kreidberg, L., Bean, J. L., Désert, J.-M., et al. 2014, ApJL, 793, L27

Lee, J.-M., Heng, K., \& Irwin, P. G. J. 2013, ApJ, 778, 97

Li, G., Gordon, I. E., Rothman, L. S., et al. 2015, ApJS, 216, 15

Line, M. R., Stevenson, K. B., Bean, J., et al. 2016, AJ, 152, 203

Lustig-Yaeger, J., Meadows, V. S., \& Lincowski, A. P. 2019, AJ, 158, 27

Macintosh, B., Graham, J. R., Ingraham, P., et al. 2014, PNAS, 111, 12661

Ment, K., Dittmann, J. A., Astudillo-Defru, N., et al. 2019, AJ, 157, 32

Miller-Ricci Kempton, E., Zahnle, K., \& Fortney, J. J. 2012, ApJ, 745, 3

Mollière, P., van Boekel, R., Bouwman, J., et al. 2017, A\&A, 600, A10

Mugnai, L., Edwards, B., \& Pascale, E. 2020, ExA, submitted

Pepe, F. A., Cristiani, S., Rebolo Lopez, R., et al. 2010, Proc. SPIE, 7735, $77350 \mathrm{~F}$

Pinhas, A., Madhusudhan, N., Gandhi, S., \& MacDonald, R. 2019, MNRAS, 482, 1485

Polyansky, O. L., Kyuberis, A. A., Zobov, N. F., et al. 2018, MNRAS, 480,2597
Rauer, H., Aerts, C., Cabrera, J. \& PLATO Team 2016, AN, 337, 961

Ricker, G. R., Winn, J. N., Vanderspek, R., et al. 2014, JATIS, 1, 014003

Rocchetto, M., Waldmann, I. P., Venot, O., Lagage, P. O., \& Tinetti, G. 2016, ApJ, 833, 120

Rothman, L., Gordon, I., Barber, R., et al. 2010, JQSRT, 111, 2139

Rothman, L. S., \& Gordon, I. E. 2014, Status of the HITRAN and HITEMP Databases, Zenodo, doi:10.5281/zenodo.11207

Sing, D. K., Fortney, J. J., Nikolov, N., et al. 2015, Natur, 529, 59

Skidmore, W. 2015, RAA, 15, 1945

Tennyson, J., \& Yurchenko, S. N. 2012, MNRAS, 425, 21

Tennyson, J., Yurchenko, S. N., Al-Refaie, A. F., et al. 2016, JMoSp, 327,73

Tinetti, G., Drossart, P., Eccleston, P., et al. 2015, ExA, 40, 329

Tinetti, G., Drossart, P., Eccleston, P., et al. 2018, ExA, 46, 135

Tsiaras, A., Rocchetto, M., Waldmann, I. P., et al. 2016, ApJ, 820, 99

Tsiaras, A., Waldmann, I. P., Tinetti, G., Tennyson, J., \& Yurchenko, S. N. 2019, NatAs, 3, 1086

Tsiaras, A., Waldmann, I. P., Zingales, T., et al. 2018, AJ, 155, 156

Venot, O., \& Agúndez, M. 2015, ExA, 40, 469

Venot, O., Hébrard, E., Agúndez, M., et al. 2012, A\&A, 546, A43

Waldmann, I. P., Rocchetto, M., Tinetti, G., et al. 2015a, ApJ, 813, 13

Waldmann, I. P., Tinetti, G., Rocchetto, M., et al. 2015b, ApJ, 802, 107

Yurchenko, S. N., Barber, R. J., \& Tennyson, J. 2011, MNRAS, 413, 1828

Yurchenko, S. N., \& Tennyson, J. 2014, MNRAS, 440, 1649 